\title{
The Spacing Effect for Structural Synaptic Plasticity Provides Specificity and Precision in Plastic Changes
}

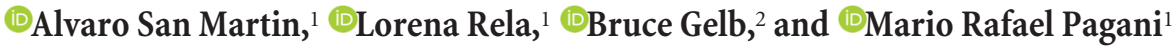 \\ ${ }^{1}$ Instituto de Fisiología y Biofísica Bernardo Houssay, Grupo de Neurociencia de Sistemas, Facultad de Medicina, Universidad de Buenos Aires, \\ The National Scientific and Technical Research Council, Buenos Aires C1121ABG, Argentina, and ${ }^{2}$ Mindich Child Health and Development Institute, \\ Departments of Pediatrics and Genetics and Genomic Sciences, Icahn School of Medicine at Mount Sinai, New York, New York 10029
}

In contrast to trials of training without intervals (massed training), training trials spaced over time (spaced training) induce a more persistent memory identified as long-term memory (LTM). This phenomenon, known as the spacing effect for memory, is poorly understood. LTM is supported by structural synaptic plasticity; however, how synapses integrate spaced stimuli remains elusive. Here, we analyzed events of structural synaptic plasticity at the single-synapse level after distinct patterns of stimulation in motoneurons of Drosophila. We found that the spacing effect is a phenomenon detected at synaptic level, which determines the specificity and the precision in structural synaptic plasticity. Whereas a single pulse of stimulation (massed) induced structural synaptic plasticity, the same amount of stimulation divided in three spaced stimuli completely prevented it. This inhibitory effect was determined by the length of the interstimulus intervals. The inhibitory effect of the spacing was lost by suppressing the activity of Ras or mitogen-activated protein kinase, whereas the overexpression of Ras-WT enhanced it. Moreover, dividing the same total time of stimulation into five or more stimuli produced a higher precision in the number of events of plasticity. Ras mutations associated with intellectual disability abolished the spacing effect and led neurons to decode distinct stimulation patterns as massed stimulation. This evidence suggests that the spacing effect for memory may result from the effect of the spacing in synaptic plasticity, which appears to be a property not limited to neurons involved in learning and memory. We propose a model of spacing-dependent structural synaptic plasticity.

Key words: Drosophila mononeurons; spaced stimulation; stimulus integration and decoding; structural synaptic plasticity

Significance Statement

Long-term memory (LTM) induced by repeated trials spaced over time is known as the spacing effect, a common property in the animal kingdom. Altered mechanisms in the spacing effect have been found in animal models of disorders with intellectual disability, such as Noonan syndrome. Although LTM is sustained by structural synaptic plasticity, how synapses integrate spaced stimuli and decode them into specific plastic changes remains elusive. Here, we show that the spacing effect is a phenomenon detected at the synaptic level, which determines the properties of the response in structural plasticity, including precision of such response. Whereas suppressing or enhancing Ras/mitogen-activated protein kinase signaling changed how synapses decode a pattern of stimuli, a disease-related Ras allele abolished the spacing effect for plastic changes.

\section{Introduction}

Activity-dependent synaptic plasticity is essential for learningdependent behavioral readjustment. It is well established that a

Received Aug. 17, 2016; revised Feb. 16, 2017; accepted April 8, 2017.

Author contributions: A.S.M., L.R., and M.R.P. designed research; A.S.M., L.R., and M.R.P. performed research; L.R., B.G., and M.R.P. contributed unpublished reagents/analytic tools; A.S.M., L.R., and M.R.P. analyzed data; A.S.M., L.R., B.G., and M.R.P. wrote the paper.

This work was supported by the National Institutes of Health (Grant R03-TW009010 to B.G.) and the National Agency of Scientific and Technological Promotion (Grant PICT-2011-2267 to M.R.P.). We thank B. Ataman, J. Ashley, and E. Belforte for technical consultations; S. Lew for data analysis with MathLab software; and E. Merlo and J. Piriz for comments on the manuscript; and members of the GNS laboratory for discussion on the experiments.

The authors declare no competing financial interests.

Correspondence should be addressed to Mario Rafael Pagani, Systems Neuroscience Section, Department of Physiology and Biophysics, School of Medicine, IFIBI0-Houssay-CONICET, 2155 Paraguay Street, University of weak training produces short-term memory based on posttranslational modifications, whereas a stronger training produces long-term memory (LTM) based on structural changes at the synaptic level (Cedar et al., 1972; Brunelli et al., 1976; Kandel et al., 1976; Castellucci et al., 1982; Bailey and Chen, 1988, 1989; Bailey et al., 1992). However, how different stimulus patterns are decoded into specific structural plastic changes is poorly understood (Kramár et al., 2012; Fujii et al., 2013).

Multiple trials of training are more effective for learning when trials are presented spaced in time (spaced learning) compared

Buenos Aires, Buenos Aires, Argentina, C1121ABG. E-mail: pagani@fmed.uba.ar or calciumsignl@gmail.com. DOI:10.1523/JNEUROSCI.2607-16.2017

Copyright $\odot 2017$ the authors $\quad 0270-6474 / 17 / 374992-16 \$ 15.00 / 0$ 
with the same amount of training without rest intervals (massed learning) (Ebbinghaus, 1885). This effect, known as the spacing effect, is a robust phenomenon that has been described extensively in humans (Ebbinghaus, 1885; Cepeda et al., 2006; Vlach and Sandhofer, 2012). In animal models, the spacing effect was detected across phyla in distinct learning paradigms including sensitization, habituation, conditioning, navigation, motor skills, and memory extinction (Ebbinghaus, 1885; Carew et al., 1972; Tully et al., 1994; Gerber et al., 1998; Hermitte et al., 1999; Beck et al., 2000; Sutton et al., 2002; Cepeda et al., 2006; Philips et al., 2007; Urcelay et al., 2009). The spacing effect is associated with LTM and the underlying mechanisms are just beginning to be elucidated. It was found that there is mitogen-activated protein kinase (MAPK) activation a few minutes after spaced or massed training (Philips et al., 2007; Pagani et al., 2009; Li et al., 2016). When two trials were in temporal proximity, as in the massed training, the second trial removed the activation of MAPK produced by the first trial. As a result, the spaced protocol produces multiple peaks of MAPK activation (one peak during every intertrial interval), whereas the massed protocol produced a single MAPK peak at the end of the protocol (Pagani et al., 2009). Temporally coordinated activity of protein kinase A (PKA) and MAPK might be required (Zhang et al., 2011). The activation of MAPK in the spaced training is required for LTM production (Philips et al., 2013). However, only the first peak of MAPK activation requires growth-factor-dependent synaptic signaling mechanisms (Kopec et al., 2015).

Although the spacing effect seems to be a fundamental principle of learning, little is known about the structural synaptic plasticity after spaced stimuli, which is expected to support longlasting behavioral changes. After massed and spaced training, reduced synaptic density correlated with the persistence of the memories produced (Aziz et al., 2014). In addition, ex vivo and in vitro studies also provide evidence of the spacing effect at synaptic level. In hippocampal slices, two trains of theta burst stimulation (TBS) spaced by $60 \mathrm{~min}$, but not $30 \mathrm{~min}$, induced a large number of dendritic spines (Kramár et al., 2012). In cultured hippocampal neurons, whereas a single high $\mathrm{K}^{+}$depolarization pulse for 3 up to $12 \mathrm{~min}$ showed no effect on filopodia formation, 4 pulses of 3 min produced a significant number of new filopodia (Wu et al., 2001). Similarly, in Drosophila larval neuromuscular junctions (NMJs), five spaced high $\mathrm{K}^{+}$stimuli rapidly produced new synaptic boutons, whereas a more massed protocol failed to produce structural synaptic plasticity (Ataman et al., 2008). These studies showed fundamental differences in structural synaptic plasticity after spaced and massed stimulation. However, it is unclear how a synapse computes and integrates spaced stimuli required for structural plastic changes.

To answer this question, we examined the effect of distinct stimulation patterns and genetic manipulations on discrete events of structural synaptic plasticity, using an ex vivo preparation of Drosophila larval NMJs, a powerful model system with which to investigate synaptic function and plasticity (Ataman et al., 2008; Koon et al., 2011; Korkut et al., 2013; Menon et al., 2013). We found a spacing-dependent structural synaptic plasticity modulation in which genetic manipulations of Ras/MAPK changed how synapses decode a stimulus pattern.

\section{Materials and Methods}

Fly stocks. Flies were raised at $29^{\circ} \mathrm{C}$ on standard cornmeal medium with 12:12 light:dark cycles and 65\% humidity. Drosophila stock UASRasV152G was generated as described previously (Oishi et al., 2006) and D42-Gal4 (RRID:BDSC_8816), UAS-mCD8.mRFP (RRID:BDSC_27398),
UAS-mCD8.mGFP (RRID:BDSC_5137), UAS-Ras85D.K (RRID:BDSC_ 5788), UAS-Ras85D.N17 (RRID:BDSC_4845), UAS-Ras85D.RNAi (RRID: BDSC_34619), and UAS-rl.RNAi (RRID:BDSC_31524) stocks were obtained from the Bloomington Drosophila Stock Center and are described at FlyBase (http://www.flybase.org).

High $\mathrm{K}^{+}$depolarization paradigm. The experimental procedure was performed as described previously (Ataman et al., 2008). Body wall muscles were dissected from males and females of wandering third instar larvae in normal-HL-3 saline (Stewart et al., 1994) containing $0.1 \mathrm{~mm}$ $\mathrm{Ca}^{2+}$. The CNS and the peripheral neurons innervating the muscle wall were left intact. Stimulations were made with high $\mathrm{K}^{+}$concentration $(90$ $\mathrm{mM}$ ), reducing $\mathrm{NaCl}$ concentration to match osmolarity of normal-HL-3 saline containing the following (in $\mathrm{mm}$ ): $40 \mathrm{NaCl}, 90 \mathrm{KCl}, 20 \mathrm{MgCl}_{2}$ $6 \mathrm{H}_{2} \mathrm{O}, 1.5 \mathrm{CaCl}_{2}, 10 \mathrm{NaCO}_{3}, 5$ sucrose, 5 trehalose, and 5 HEPES, $\mathrm{pH}$ 7.2. Chemical reagents used were from Sigma-Aldrich. Normal-HL-3 and high $\mathrm{K}^{+}$-HL-3 were interchanged to generate the different stimulation protocols.

Quantification of number and area of synaptic boutons. Live images of the NMJs were taken before and after stimulation as indicated in each protocol. Images were taken with an Olympus BX53 epifluorescence microscope equipped with an Olympus Q-Color5 digital camera using a $40 \times$ water-immersion objective. RFP-labeled nerve terminals of the larval NMJs were taken at muscles 6 and 7 of abdominal segment 3. Several high-resolution pictures $(2560 \times 1920$ pixels $)$ were obtained at different levels of the $z$-axis to include the whole nerve terminal. Then, images were merged using method $\mathrm{C}$ (pyramidal) in Focus version 6.0.18 software (Helicon), which provided the final image. New synaptic boutons were identified as rounded synaptic processes present in images after stimulation but absent before stimulation. The total number of synaptic boutons was quantified in the images obtained before stimulation. The area of new synaptic boutons was measured after defining ROIs for boutons without including the neck with ImageJ version 1.49. Measures were obtained in pixels for each synaptic bouton.

Plasma membrane integrity assay. Dissections of the NMJ preparations were performed as described above. Four hours after the stimulation conditions (i.e., 16 min of massed stimulation or nonstimulated control), NMJ preparations were incubated with propidium iodide $(1 \mu \mathrm{g} /$ $\mathrm{ml}$ ) for $30 \mathrm{~min}$. The nonstimulated control was incubated with HL-3 containing $0 \mathrm{~mm} \mathrm{Ca}^{2+}$ and EGTA $1 \mathrm{~mm}$. Live images were obtained after staining.

Electrophysiology. Larvae were dissected and subjected to the high $\mathrm{K}^{+}$ depolarization protocols as indicated above. Recordings were performed in HL-3 saline (Stewart et al., 1994) containing $0.5 \mathrm{~mm}$ calcium at room temperature as described previously (Ataman et al., 2008). Body wall muscles were visualized under a Nikon FN1 microscope equipped with epifluorescence illumination using a $40 \times$ water-immersion longworking distance objective (numerical aperture: 0.8 ; working distance: $2 \mathrm{~mm}$ ). Body wall muscles 6 and 7 of abdominal segment 3 were impaled at $\sim 50 \mu \mathrm{m}$ from motoneuron terminals identified by fluorescence with borosilicate glass (inner diameter: $0.58 \mathrm{~mm}$, outer diameter: $1 \mathrm{~mm}$ ) (WPI catalog \#1B100F-4) microelectrodes (25-40 M $\Omega$ ) shaped with a puller (P-97; Sutter Instruments) and filled with $3 \mathrm{M} \mathrm{KCl}$. Signals were amplified using a Multiclamp 700B amplifier (Molecular Devices) in currentclamp mode, low-pass filtered at $200 \mathrm{~Hz}$, digitized at $20-25 \mathrm{kHz}$ (Digidata 1200; Molecular Devices), and acquired on a PC running pClamp 9 software (Molecular Devices). Only muscle cells with a resting potential of at least $-50 \mathrm{mV}$ and input resistance of at least $3 \mathrm{M} \Omega$ were used for analysis. Input resistance was determined from the voltage deflection produced by a $-1 \mathrm{nA}, 500 \mathrm{~ms}$ current pulse. Miniature excitatory junction potential $(\mathrm{mEJP})$ frequency was calculated from the total count of events observed in $120 \mathrm{~s}$ recordings using Clampfit software (Molecular Devices). Counts were done by an experimenter blinded to treatments. Data were normalized to the average frequency of nonstimulated controls.

Crawling activity assay. Temperature-induced crawling assay was performed as described previously (Tsai et al., 2012). Crawling activity was measured form third instar larvae raised at $29^{\circ} \mathrm{C}$. Experiments where performed at room temperature set at $29^{\circ} \mathrm{C}$ and $70 \%$ humidity. Larvae were placed in Petri dishes of $8 \mathrm{~cm}$ diameter containing agar $1.8 \%$ stained dark blue for better contrast. Larvae were recorded with a webcam for a 
period of $10 \mathrm{~min}$ (30 Hz sampling). Automated tracking was made with Any-Maze version 5.14 software. Immobility was estimated as the persistence of the position of the body of the animal ( $65 \%$ of the body or more) for a period $\geq 4 \mathrm{~s}$.

Statistical analysis. Statistical analyses were performed with Prism version 6.01 software (GraphPad) except for bootstrapping resampling and Monte Carlo permutation test, which were performed using MATLAB version 8.6.0.267246 (R2015b), and the likelihood ratio test for overdispersion, which was performed using $\mathrm{R}$ version 3.3.1. Statistical analysis was performed after quantification of new synaptic boutons on highresolution recorded images. The Shapiro-Wilk test was used to evaluate data normality and Bartlett's test for homogeneity of variances. For new synaptic boutons, nonparametric tests were used comparing multiple groups with Kruskal-Wallis test followed by Dunn's multiplecomparisons test. For total number of boutons one-way ANOVA test was used.

\section{Results}

\section{Spaced, but not pseudo-massed, stimuli induce structural} synaptic plasticity

To investigate how specific stimulus patterns turn into specific events of synaptic plasticity, we used an ex vivo NMJ preparation expressing a membrane-bound RFP (mCD8-RFP) in motoneurons. We began using specific protocols of stimulation with high $\mathrm{K}^{+}$described and characterized previously (Ataman et al., 2008). In that study, 16 min of total stimulation was presented in two distinct patterns, the spaced protocol, in which the stimulation was distributed in five pulses spaced by $15 \mathrm{~min}$, and the pseudomassed protocol distributed in three pulses spaced by $15 \mathrm{~min}$. In contrast to the pseudo-massed protocol, the spaced protocol produced new synaptic boutons, rounded synaptic processes with delayed formation of postsynaptic specialization characterized previously in detail (Ataman et al., 2008; Korkut et al., 2013; Piccioli and Littleton, 2014). Moreover, a more physiological stimulation (nerve stimulation or light-activated channel rhodopsin-2 in the intact animal) produces a similar effect (Ataman et al., 2008).

We used the motoneuron-specific driver D42-GAL4 to drive the expression of the UAS-RFP (Sanyal, 2009) and imaged nerve terminals before and after stimulation. We began examining the synaptic plasticity response after the spaced and pseudo-massed stimulation. Consistent with the original report, the spaced protocol produced a significant number of new synaptic boutons 40 min after stimulation, whereas the pseudo-massed protocol did not (Fig. $1 A-D$ ). The pseudo-massed protocol did not produce new synaptic boutons even 74 min after the stimulation, which covers the time required in the spaced protocol (Fig. 1D). The structural synaptic plasticity was an activity-dependent phenomenon because, in the absence of stimulation, we did not observe new synaptic boutons (Fig. 1D). The pseudo-massed protocol effectively provides a more massed stimulation than the spaced one, which led to the conclusion that the rapid induction of structural synaptic plasticity requires spaced stimulation $(\mathrm{Wu}$ et al., 2001; Ataman et al., 2008).

\section{Activity-dependent synaptic plasticity after massed stimulation}

It is important to note that the pseudo-massed stimulation protocol contains two interstimulus intervals (ISIs) of $15 \mathrm{~min}$ (Fig. $1 A)$. Therefore, this protocol is not truly a massed stimulation, which makes it difficult to interpret the effect of this protocol on synaptic plasticity compared with the spaced one. To develop a truly massed protocol of stimulation, we examined the effect of 16 min of continuous stimulation. First, we confirmed that this strong protocol did not damage the NMJ preparation. To do so, we performed a plasma membrane integrity assay (see Materials and Methods). Then, to detect signs of damage, we also examined: the nerves of motoneurons after $4 \mathrm{~h}$ of nonstimulation (NS) and M16' stimulations to detect varicosities or discontinuities (Xiong and Collins, 2012) and the NMJ morphology compared with the initial state (data not shown). In addition, electrophysiological experiments also support that the M16' protocol does not damage the NMJ (see below).

Unexpectedly, the massed protocol generated as many new synaptic boutons as the spaced protocol $40 \mathrm{~min}$ after stimulation or 64 min later, which covers the time required in the spaced protocol (Fig. $1 A, D, E$ ). There was no difference in the size of the new synaptic boutons produced by the spaced and the massed protocols (data not shown). A straightforward interpretation is that, when a single stimulation pulse is sufficiently long, synaptic plasticity is also possible. This implies that each of the pulses in the pseudo-massed protocol (i.e., 5 or 6 min; Fig. 1A) individually should be unable to produce new synaptic boutons. Therefore, we examined the effect of massed stimulation of increasing duration from 2 up to $16 \mathrm{~min}$. These experiments revealed two properties. First, a significant number of new synaptic boutons can be generated by a single pulse of stimulation as short as $6 \mathrm{~min}$ (Fig. $1 F$ ). This evidence indicates that the absence of new synaptic boutons after the pseudo-massed protocol cannot be explained by a short duration of its individual pulses of stimulation. We investigated in detail this paradoxical synaptic property below. Second, the number of new synaptic boutons produced by a single pulse of stimulation showed a sigmoid relationship with the duration of stimulation, suggesting cooperative mechanisms mediating these plastic changes (Fig. 1F; Salazar et al., 2010).

The formation of new synaptic boutons by a single pulse of stimulation provides an intriguing situation. How is it possible that a single pulse of 6 min produced new synaptic boutons, whereas a pseudo-massed protocol containing pulses of 5 and 6 min did not? This cannot be explained by the time at which the picture was taken because a second picture $120 \mathrm{~min}$ after the beginning of the stimulation showed the same result (Fig. $1 A, D, F)$. One possible explanation emerges after an analysis of the differences among protocols (Fig. 2A). The pseudo-massed protocol contains two ISIs, whereas the massed does not (Fig. $2 A$ ). Therefore, the ISI may produce a negative effect on the generation of new synaptic boutons, which precludes plasticity in the pseudo-massed protocol. However, in the spaced protocol, the inhibitory effect on plasticity might be overcome by the additional repetition of stimulation (two additional pulses compared with the pseudo-massed protocol; Fig. 2A). To test this possibility, we performed experiments presented in the following two sections.

\section{ISI-dependent inhibition of synaptic plasticity in the pseudo-massed protocol}

The fact that a single pulse of 6 min induced plasticity (Fig. $1 F$ ) but the pseudo-massed protocol with a last pulse of $6 \mathrm{~min}$ did not indicated that the inhibitory effect of the ISI may be an active effect, not a passive one. It should be noted here that, during an ISI, mechanisms activated by the preceding pulse of stimulation are expected to occur. We rationalized that the inhibitory effect of the ISI should have a proper duration to be effective, which can be evident by shortening the ISI. Therefore, if the length of the ISI is not effective after shortening, the pulses will be decoded as a massed stimulation. To test this idea, we examined the effect of a pseudo-massed protocol with an ISI of 10, 15, or 20 min duration. Whereas a pseudo-massed protocol containing two ISIs of 
A
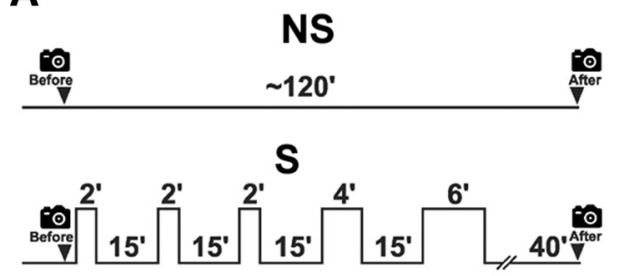

\section{PM}
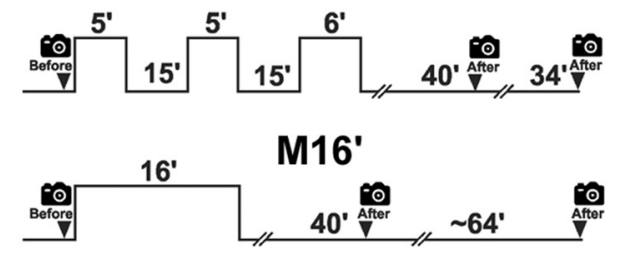

\section{C}
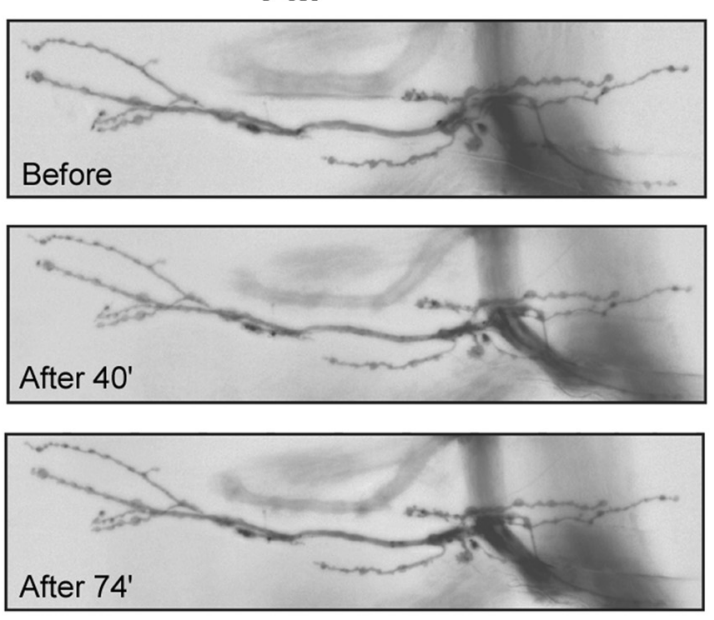

$\mathbf{E}$

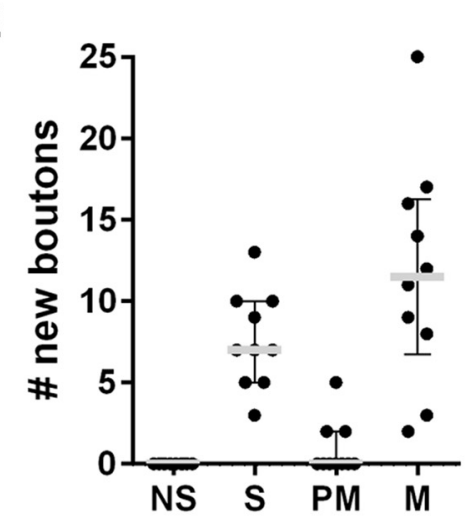

B

$\mathbf{S}$
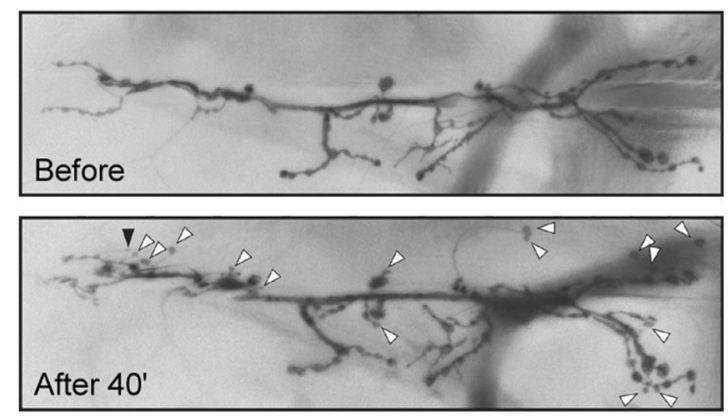

D
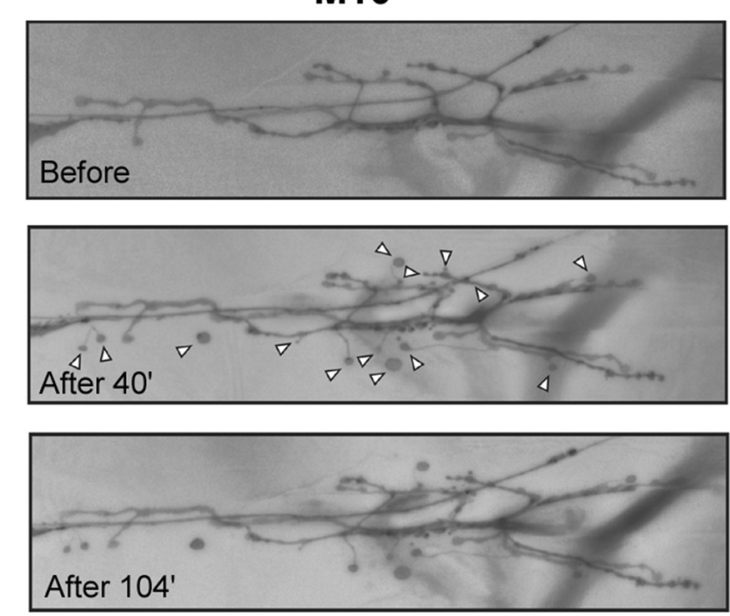

Figure 1. Spaced or massed, but not pseudo-massed, stimulation induces new synaptic boutons. A, Protocols of stimulation. Control nonstimulated (NS), spaced stimulation (S), pseudo-massed stimulation (PM), and massed stimulation (M16'). The time of image recoding is represented by a camera icon. The protocol architecture reproduces a previous report (Ataman et al., 2008). $B$, Representative motor nerve terminal expressing membrane-tethered RFP imaged at the larval NMJ before and 40 min after the spaced stimulation protocol (S). White arrowheads indicate new synaptic boutons and black arrowhead indicates a new filopodium-like structure. $C$, NMJ before and after stimulation with a PM protocol at the time points indicated in the protocol above. D, Quantification of new synaptic boutons $40 \mathrm{~min}$ after stimulation conditions presented in $\boldsymbol{A}$. Scatter dot plot showing median (gray line) with interquartile range. Black dots represent each replicate. $n=10 \mathrm{NMJs}$ from five animals. Asterisks indicate a significant difference compared with control NS; $S p=0.0004$ and M16' $p<0.0001$; Kruskal-Wallis test followed by Dunn's multiple-comparisons test. $\boldsymbol{E}$, NMJ before and after stimulation with a massed (M16') protocol at the time points indicated in the protocol above. $F$, New synaptic boutons after massed stimulation of increasing duration. Sigmoidal fit, with confidence interval (dot line), $n=54$, each time point $9 \mathrm{NMJs}$ from 5 animals, $R^{2}=0.4789$. Black dots represent each replicate. Empty circles represent means. *Significant difference after 6 min of massed stimulation compared with control NS ( $p=0.0074$, Kruskal-Wallis test followed by Dunn's multiple-comparisons test). 
A

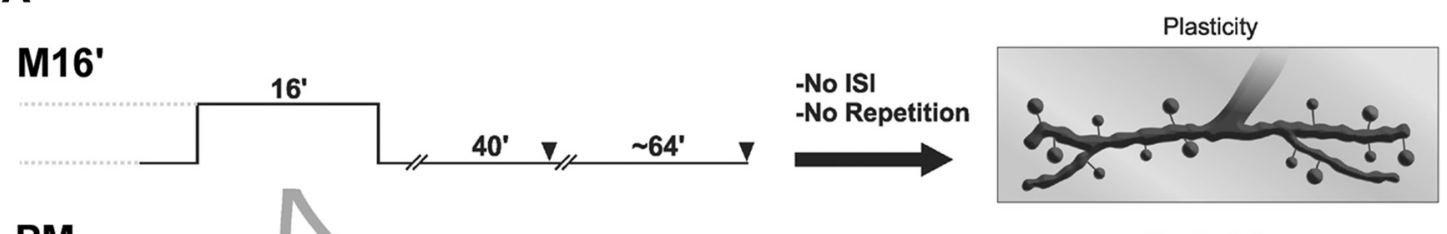

PM
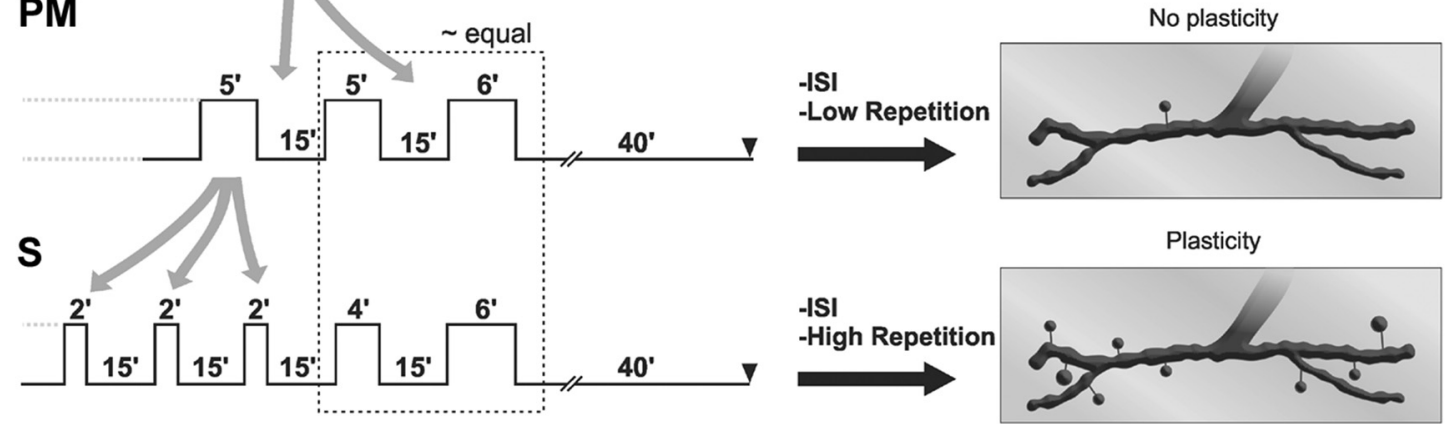

B

PM ISI10'

C
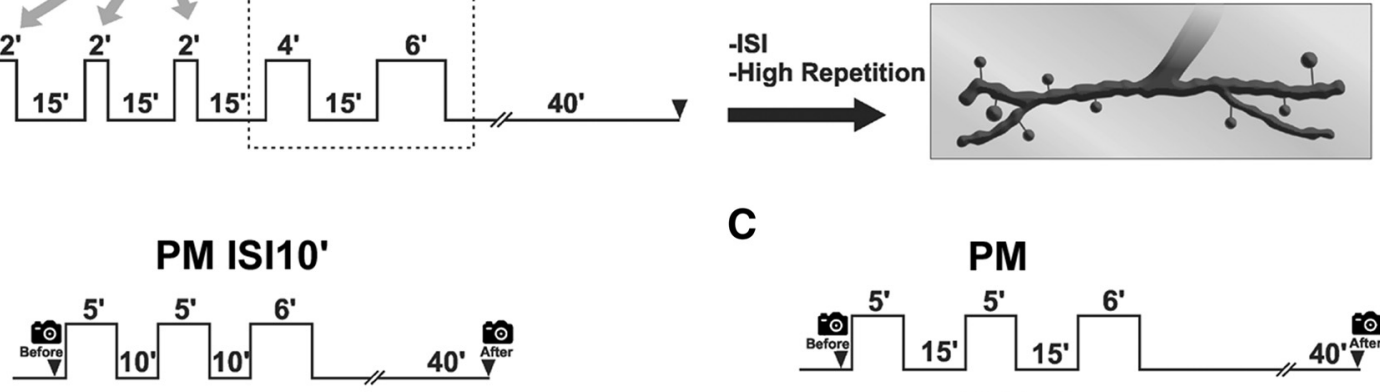

PM

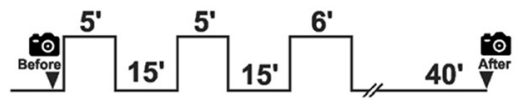

PM ISI20'
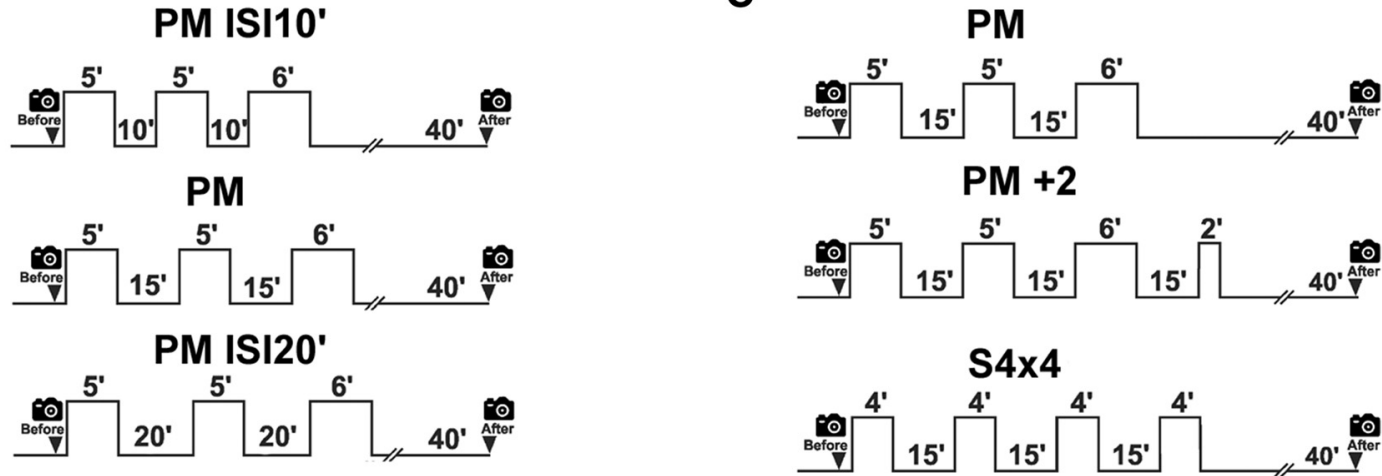

\section{PM +2}
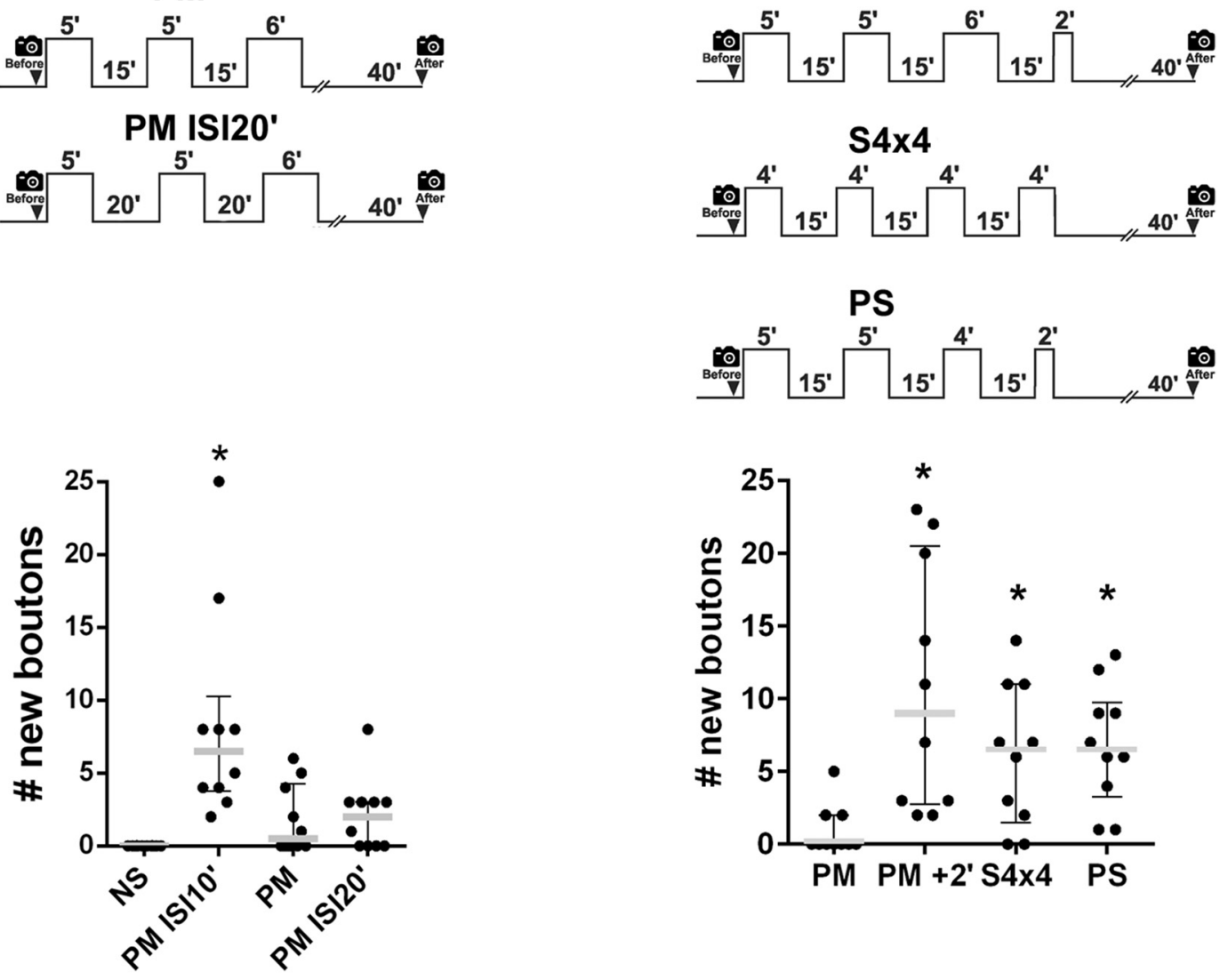

Figure 2. Effect of the spacing and the repetition in stimuli regulates structural synaptic plasticity. A, Comparison of protocols aimed at understanding why the pseudo-massed stimulation did not produce synaptic plasticity. Schematic representation of protocols (left) and their responses in plasticity (right) are shown. Whereas the massed (M16') and the spaced protocol (S) induced synaptic plasticity, the pseudo-massed (PM) did not. In contrast to the M16' protocol, the PM protocol contains two ISIs (two gray arrows), which might inhibit plasticity. Comparing the PM and the $S$ protocol, the last two pulses are similar. However, in contrast to the PM protocol, the S protocol contains additional pulses of stimulation (three gray arrows), which might promote plasticity. $\boldsymbol{B}$, Top, Protocols of pseudo-massed stimulation with ISIs of $10 \mathrm{~min}$ (PM ISI10'), the standard interval of $15 \mathrm{~min}$ (PM), and $20 \mathrm{~min}$ (PM ISI20'). Bottom, Quantification of new synaptic boutons 40 min after stimulation. Scatter dot plot showing median (gray line) with interquartile range. Black dots represent each replicate. $n=10 \mathrm{NMJs}$ from 5 animals. *Significant difference compared with control NS; PM $p<0.0001$; Kruskal-Wallis test followed by Dunn's multiple-comparisons test. C, Top, Protocols of stimulation. Bottom, Quantification of new synaptic boutons 40 min after stimulation. Scatter dot plot showing median (gray line) with interquartile range. Black dots represent each replicate. $n=10 \mathrm{NMJs}$ from 5 animals. *Significant difference compared with PM; PM+2' $p=$ $0.0007 ; S 4 \times 4 p=0.0286 ; \mathrm{PS} p=0.0072 ;$ Kruskal-Wallis test followed by Dunn's multiple-comparisons test. 
15 or 20 min did not generate new synaptic boutons, the same protocol with $10 \mathrm{~min}$ of ISIs produced a large number of boutons (Fig. 2B), similar to the massed protocol containing $16 \mathrm{~min}$ of stimulation (Fig. 1E). This implies the existence of underlying processes during the ISI, an aspect that we explore later. This recall the molecular mechanisms during the intertrial interval required for the spacing effect in a context of memory (Pagani et al., 2009; Philips et al., 2013; Kopec et al., 2015).

\section{Repetition effect overcomes the inhibitory effect of the ISI in the spaced protocol}

We analyzed whether the additional pulses of stimulation in the spaced protocol overcome the inhibitory effect of the ISI detected in the pseudo-massed protocol. To test the existence of this repetition effect, we first examined the effect of the addition of a single 2 min pulse of stimulation to the pseudo-massed protocol with the standard ISI of $15 \mathrm{~min}$ (Fig. 2C). Note that a small pulse of $2 \mathrm{~min}$ did not produce synaptic plasticity by itself (Fig. $1 F$ ). This extended protocol of four pulses produced a significant number of new synaptic boutons, supporting the notion that additional pulses of stimulation can overcome the inhibitory effect in the pseudo-massed protocol (Fig. 2C). However, this strategy was not enough to discriminate the effects of adding a pulse from the increase in the total amount of stimulation because the total time of stimulation in this extended protocol was $18 \mathrm{~min}$ $(16+2 \mathrm{~min})$ instead of $16 \mathrm{~min}$. Therefore, we rearranged the duration of the pulses to keep a total time of $16 \mathrm{~min}$. The pseudomassed protocol with pulses of 5, 5, and 6 min was reorganized into two new protocols: 4, 4, 4, 4 min (named "S4×4") and 5, 5, 4, 2 min (named "positively skewed") (Fig. 2C). Note that, in the latter protocol, the duration of successive pulses was reduced, in contrast to the spaced protocol, whereas the $S 4 \times 4$ protocol was symmetric. Both rearranged protocols produced a significant number of new synaptic boutons (Fig. 2C), supporting the idea of a repetition effect overcoming the inhibitory effect of the ISI. These results also indicated that neither the asymmetry nor the longer pulses at the end are needed for synaptic plasticity in the standard spaced protocol (a property that we make use below). It was shown previously that 4 , but not 3, pulses of $2 \mathrm{~min}$ also induced structural synaptic plasticity in Drosophila NMJ and in cultured hippocampal neurons (Wu et al., 2001; Ataman et al., 2008). Altogether, these data suggest that the fourth pulse overcomes the inhibitory effect found in the three-pulse protocol independently of the size of the pulses and the total time of stimulation.

Altogether, these observations suggest the integration of two effects in the spaced protocol: the spacing effect, which is inhibitory to the formation of new synaptic boutons, and the repetition effect, which promotes the formation of new synaptic boutons after more than three pulses of stimulation. Therefore, we conclude that, in the pseudo-massed protocol, the inhibition exerted by the ISI precluded synaptic plasticity. However, when the intervals between stimuli are excessively short (i.e., $\leq 10 \mathrm{~min}$ ), there is no inhibition mediated by the ISI and the synapses decoded this pattern as a massed stimulation.

\section{Ras/MAPK controls the spacing effect in structural synaptic plasticity}

To gain insights into the mechanisms of the synaptic phenomena described above and to provide further support for our interpretations, we looked for molecular components that could act during the ISI. Previously, we showed that the phosphatase corkscrew modulates MAPK activity during the intertrial intervals of a spaced protocol of olfactory learning, presumably through the Ras/MAPK pathway (Pagani et al., 2009). Moreover, MAPK appears to be differentially regulated during spaced and massed stimulation and its inhibition or inhibition of Ras in hippocampal neurons prevents long-term potentiation (LTP) and spine enlargement (Wu et al., 2001; Harvey et al., 2008). Finally, because Ras regulates the number of synaptic boutons in developing fly NMJ upstream of PI3K, Ral and Raf (Koh et al., 2002), we analyzed the effect of suppressing or enhancing Ras activity on the formation of new synaptic boutons after stimulation.

First, we examined the effect of reducing Ras activity in motoneurons by expressing the dominant-negative allele $\mathrm{Ras}^{\mathrm{N} 17}$ (UAS-Ras.N17/+;CD8.mRFP/+;D42/+). Surprisingly, Ras ${ }^{\mathrm{N} 17}$ had no effect on the number of new synaptic boutons after the spaced protocol compared with the control genotype $(+/+$; CD8.mRFP;D42/+; Fig. 3A). However, the inhibition of Ras promoted a significant number of new synaptic boutons after the pseudo-massed protocol compared with the control genotype $(+/+$; CD8.mRFP; D42/+) or control stimulation (NS) (Fig. $3 A$ ). A similar result was obtained by using a Ras.RNAi (Fig. 3B). This evidence suggested that Ras participates in the inhibition mediated by the ISI because the inhibitory effect of the pseudomassed protocol disappeared in the presence of a Ras dominantnegative allele.

Second, we examined the effect of enhancing Ras activity in motoneurons by overexpressing a WT allele Ras WT (CD8.mRFP/ UAS-Ras.WT;D42/+). Consistent with the other data, Ras WT had no effect on the number of new synaptic boutons after the pseudo-massed protocol compared with the control genotype $(+/+$; CD8.mRFP; D42/+) (Fig. 3C). However, the overexpression of Ras prevented synaptic plasticity after the spaced stimulation (Fig. 3C), suggesting that the inhibitory effect produced by $\operatorname{Ras}^{\mathrm{WT}}$ cannot be overcome by five pulses of stimulation. Therefore, activity levels of Ras can determine how the synapse decodes a stimulus pattern into distinctive plastic changes.

Next, we investigated whether the suppression of rolled, the Drosophila ortholog of the MAPK ERK1/2 (Biggs et al., 1994), produced an effect on synaptic plasticity similar to the dominantnegative Ras.N17. Reducing rolled activity in motoneurons by expressing an rl-RNAi (CD8.mRFP/+;D42/UAS-rl-RNAi) had no effect in plasticity after spaced stimulation and promoted a significant plasticity after the pseudo-massed protocol (Fig. 3D), suggesting that Ras/MAPK signaling controls the spacing effect on synaptic plasticity.

Although it seems clear that the ISI produces an inhibitory effect and Ras mediates an inhibitory effect on synaptic plasticity as well (Figs. 2, 3A-C), the evidence presented did not support that Ras acts in an ISI-dependent manner. If Ras inhibits the formation of new boutons during the ISI, then genetic manipulations of Ras should have no effect on synaptic plasticity after massed stimulation because there was no ISI in this protocol. We tested this prediction and found that neither suppressing nor enhancing Ras activity produced a significant effect on the number of new synaptic boutons after massed stimulation (Fig. 3E). Similarly, suppressing rolled had no effect on plasticity after massed stimulation (Fig. 3E).

Motoneurons expressing Ras or rolled transgenes before stimulation showed a reduced number of synaptic boutons compared with the control genotype. However, the number of synaptic boutons before stimulation in motoneurons with enhanced or suppressed activity of Ras/MAPK was the same (data not shown), in agreement with previous reports (Koh et 
A
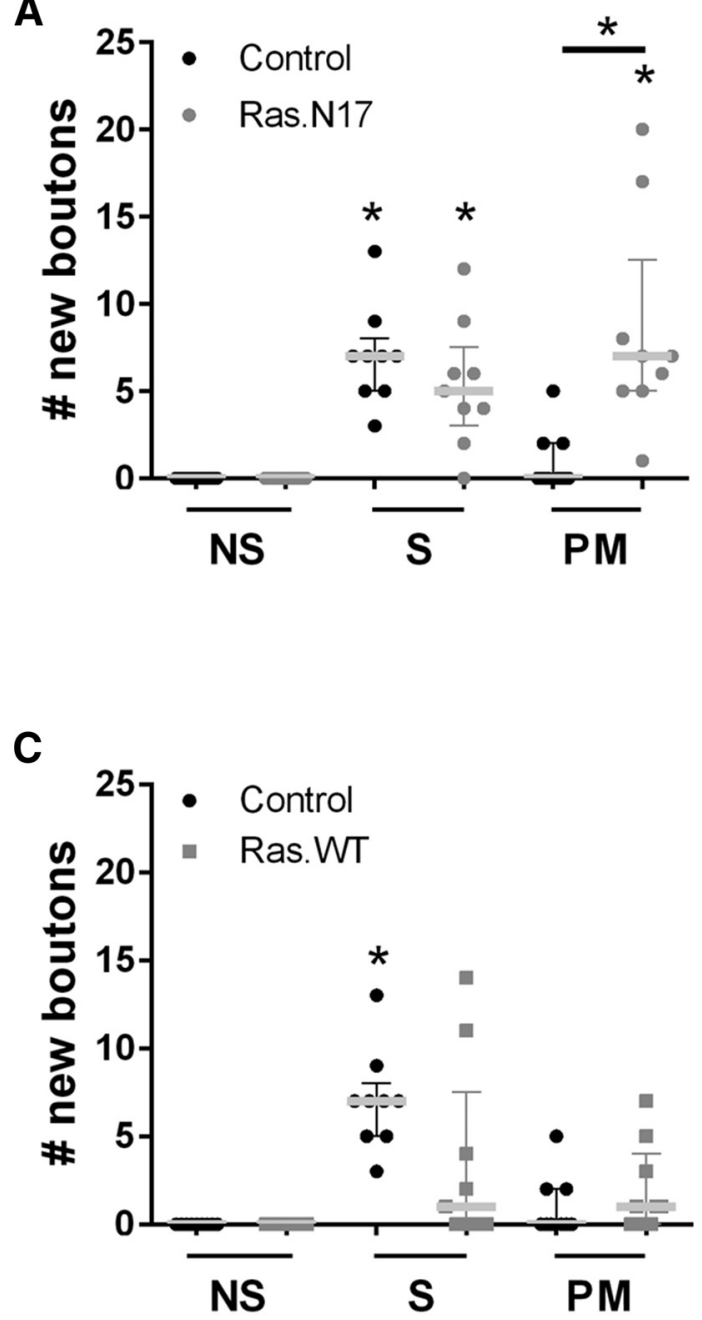

B

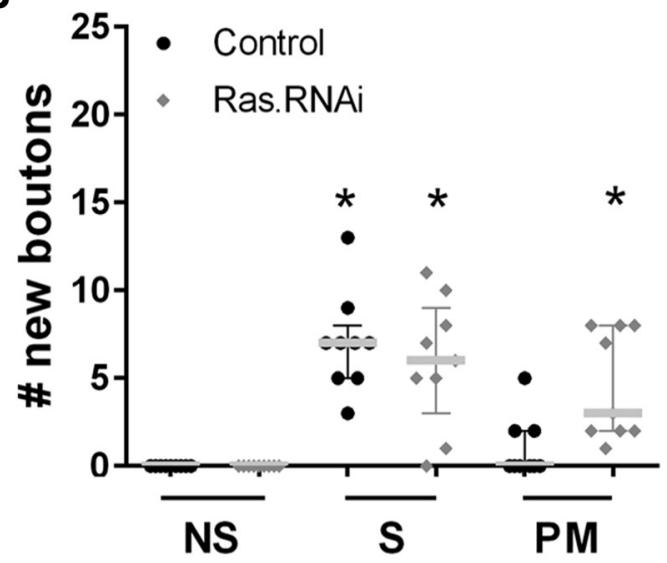

D

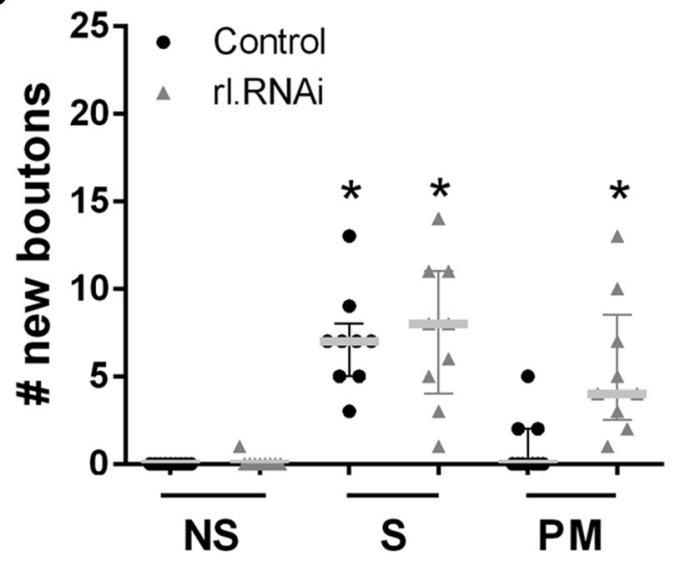

ns

E

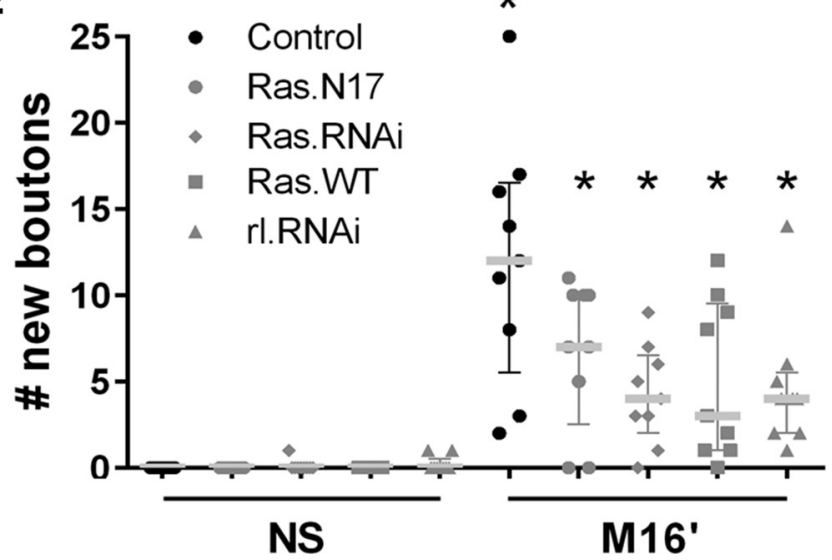

Figure 3. Ras/MAPK mediates the ISI-dependent inhibition in synaptic plasticity. $\boldsymbol{A}-\boldsymbol{D}$, Quantification of new synaptic boutons 40 min after stimulation conditions: Control nonstimulated (NS) compared with spaced (S) and pseudo-massed stimulation (PM) in control genotype and Ras.N17 genotype $(\boldsymbol{A})$, Ras.RNAi $(\boldsymbol{B})$, Ras.WT genotype $(\boldsymbol{C})$, and rl.RNAi genotype (D). $n=9$ NMJ from 5 animals. $\boldsymbol{A}$, Control genotype: NS versus $S, p=0.0017$; Ras.N17 genotype: NS versus $S, p=0.0205$ and NS versus PM, $p=0.0017 ;$ PM stimulation: control genotype versus Ras.N17, $p=0.0462$. $\boldsymbol{B}$, Control genotype: NS versus $S, p=0.0005$; Ras.RNAi genotype: NS versus $S, p=0.0009$; and NS versus PM $p=0.02$. C, Control genotype: NS versus $S, p=0.0017$. $\boldsymbol{D}$, Control genotype: NS versus $S, p=0.0017$; rl.RNAi genotype: NS versus $S, p=0.003$; and NS versus PM, $p=0.0365$, Kruskal-Wallis test followed by Dunn's multiple-comparisons test. For $\boldsymbol{A}-\boldsymbol{C}$, a single test was made including all the data. $\boldsymbol{E}$, Quantification of new synaptic boutons 40 min after stimulation conditions: Control NS compared with massed stimulation (M16') in control genotype and Ras.N17, Ras.RNAi, Ras.WT, and rl.RNAi genotypes. $n=9$ NMJs from 5 animals. Scatter dot plot showing median (gray line) with interquartile range. *Significant difference NS versus M16', control genotype, $p<0.0001$; Ras.N17, $p=0.0071$; Ras.RNAi, $p=0.0346$; Ras.WT, $p=0.0136$; and rl.RNAi, $p=0.0351$, Kruskal-Wallis test followed by Dunn's multiple-comparisons test. 
A

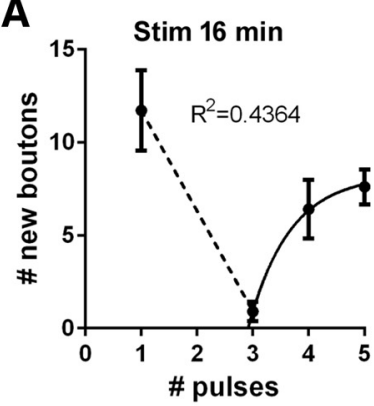

D

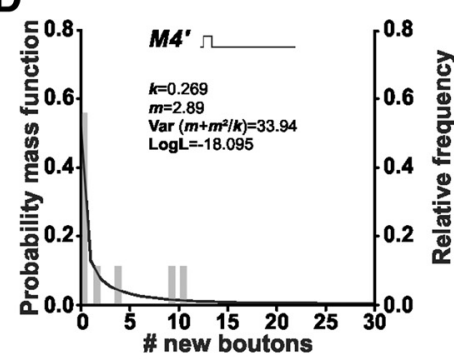

B

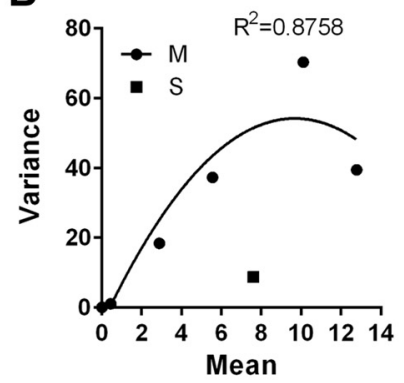

E

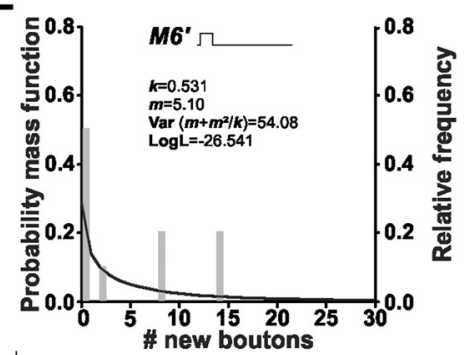

C

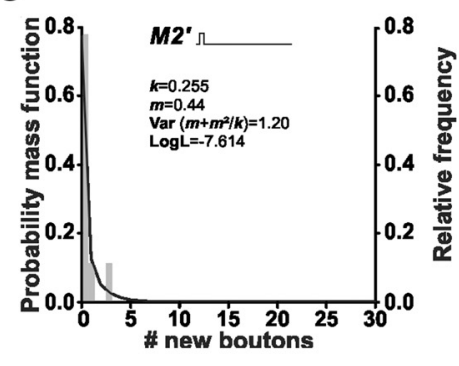

F

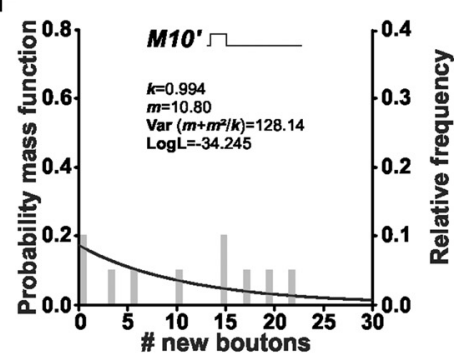

G

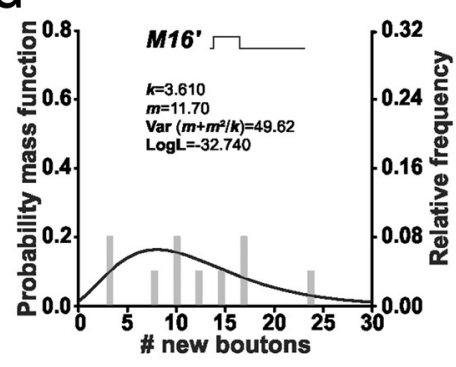

J

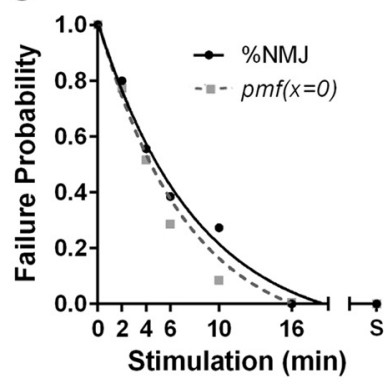

M

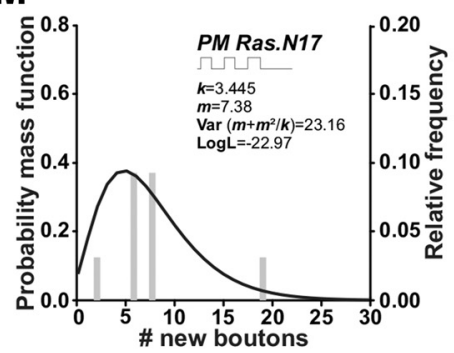

H

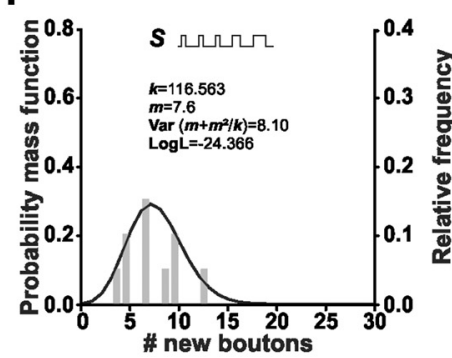

K

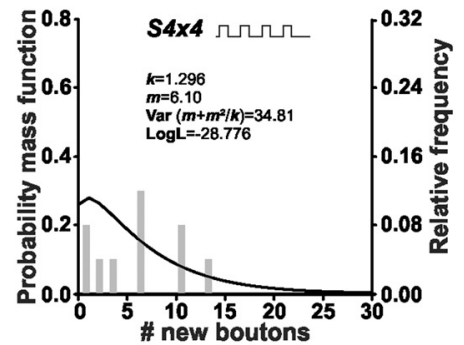

N

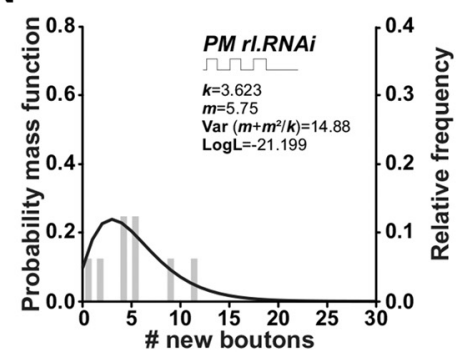

I

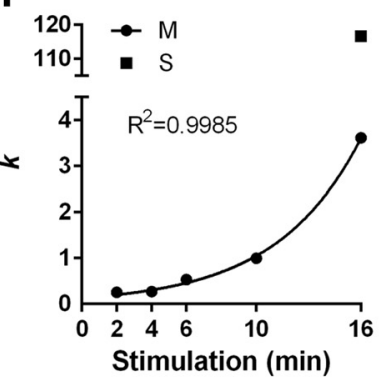

L

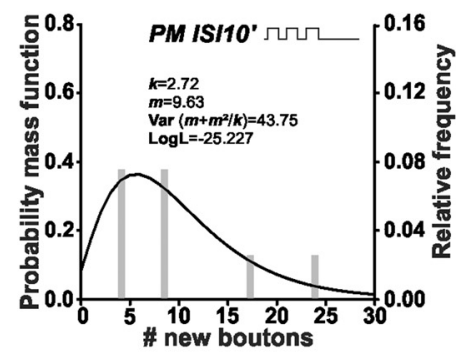

Figure 4. Spaced stimulation reduced ambiguous synaptic responses in plasticity. $A$, Number of new synaptic boutons versus number of pulses in protocols totaling 16 min of stimulation. Exponential one phase decay fit for three or more pulses. $n=10 \mathrm{NMJs}$ from 5 animals for each number of pulses. $\boldsymbol{B}$, Variance of the number of new synaptic boutons as a function of the mean after a single pulse of increasing durations $0,2,4,6,10$, and $16 \mathrm{~min}(\mathrm{M})$ with quadratic fit and after spaced stimulation (S) with a total time of $16 \mathrm{~min}, n=9 \mathrm{NMJ}$ from 5 animals for each mean. (C-H) Frequency distribution of new synaptic boutons showing overlapped a nonlineal regression fit to a negative binomial model after massed stimulation of increasing duration: 2 min (M2') (C), 4 min $\left(M 4^{\prime}\right)(\boldsymbol{D}), 6 \mathrm{~min}\left(\mathrm{MG}^{\prime}\right)(\boldsymbol{E}), 10 \mathrm{~min}\left(\mathrm{M} 10^{\prime}\right)(\boldsymbol{F}), 16 \mathrm{~min}\left(\mathrm{M} 16^{\prime}\right)(\boldsymbol{G})$, and after the spaced (S) protocols of $16 \mathrm{~min}$ of total stimulation (H), in each case $n=9$ NMJs (Figure legend continues.) 
al., 2002), indicating that the effects reported above (Fig. $3 A-D)$ were activity dependent.

Together, these results showed that overexpressing Ras precluded the synaptic plasticity induced by the spaced protocol. Moreover, new synaptic boutons can be detected by reducing Ras or MAPK activity (Fig. $3 A-D$ ) even after the pseudo-massed protocol, which is insufficient to promote plasticity in the control genotype. In other words, Ras/MAPK is essential for the inhibitory effect of the ISI because Ras/MAPK manipulations had no effect in the absence of ISIs (Fig. 3E). These results further supported the existence of an inhibitory effect mediated by the ISI in the pseudo-massed protocol.

Ras/MAPK seems to only regulate the formation of new synaptic boutons but does not mediate their formation because new boutons can be formed even with an effective reduction after the spaced protocol (Fig. $3 A, B, D$ ).

\section{Spaced stimuli promote precision in structural synaptic plasticity}

What would be the role of the ISI-dependent inhibition and the repetition effect? In contrast to a single pulse of $16 \mathrm{~min}$ (massed stimulation), $16 \mathrm{~min}$ of stimulation divided in three pulses spaced by 15 min prevented structural plasticity (Fig. 4A). An exponential recovery was observed by dividing the $16 \mathrm{~min}$ of stimulation in four and five pulses (Fig. 4A). This recovery was associated with a reduced dispersion after five pulses of stimulation, suggesting a change in data distribution induced by the spaced protocol. To address this possibility, we analyzed the properties of the data produced by massed and spaced stimulation.

The variance of the number of new synaptic boutons after massed stimulation was larger than the mean (variance $>$ mean, which is known as overdispersion; Bliss and Fisher, 1953; Lindén and Mäntyniemi, 2011), increasing with the duration of the pulse up to $10 \mathrm{~min}$, but became smaller (more precise) after that (Fig. $4 B$ ). This variability seems to be even smaller after spaced stimulation (variance $=$ mean) $($ Fig. $4 B)$. We used the negative binomial distribution, which can model count data with both types of mean-variance relationship (Bliss and Fisher, 1953). This distribution is described by two parameters, the mean $(m)$ and the dispersion parameter $(k)$. The distribution approaches a Poisson distribution because $k$ tends to infinity, with variance $=m$, (Var $\left.=m+m^{2} / k\right)$ (Bliss and Fisher, 1953).

This model described well all the data produced by massed and spaced stimulation (Fig. 4C-H). The data produced by massed stimulation was characterized by an asymmetric distribution with small $k$ value and low probability of success in pulses shorter than $16 \mathrm{~min}$ (Fig. 4C-G,I). The probability of failure estimated as the probability mass function when $x=0$, was essentially identical to the direct observation of the NMJs showing

\section{$\leftarrow$}

(Figure legend continued.) from 5 animals. $I$, Parameter of the negative binomial fit $(k)$ as a function of the stimulation time for a single pulse from 2-16 $\mathrm{min}(\mathrm{M})$ and the standard spaced protocol (S). J, NMJs failing to produce synaptic plasticity as a function of the stimulation time (exponential one phase decay fit) after massed protocols (from 0 up to $16 \mathrm{~min}$ ) and after the standard spaced stimulation (S) totaling 16 min. Probability was estimated as follows: (\%NMJ) number of NMJs showing an absence of new synaptic boutons normalized to the number of NMJ sstimulated (failures/all trials) and $(\operatorname{pmf}(x=0))$ probability mass function of the negative binomial distributions fits when $x=0 . n=9$ NMJs from 5 animals for each stimulation time. Curves do not differ significantly, extra sum-of-squares $F$ test, $p=0.2203$. $\boldsymbol{K}-\boldsymbol{N}$, Frequency distribution of new synaptic boutons with fit to a negative binomial model after $S 4 \times 4(\boldsymbol{K})$ and PMISI10' $(\boldsymbol{L})$ in control genotype and after PM stimulation in Ras.N17 $(\boldsymbol{M})$ and rl.RNAi $(\boldsymbol{N}) . n=9$ NMJs from 5 animals. an absence of plasticity (Fig. $4 J$ ). In contrast, the data produced by spaced stimulation was characterized by a symmetric distribution with large $k$ value and a high probability of success (Fig. $4 H-J)$. The variability in events of synaptic plasticity, estimated as variance $\left(m+m^{2} / k\right)$, was 6.13 times larger after massed than spaced stimulation (Fig. 4G,H), indicating that the spaced stimulation composed of 5 pulses induced a more precise response in synaptic plasticity. In this context precision refers to how close the number of new synaptic boutons from different samples are to each other. Whereas $16 \mathrm{~min}$ of massed stimulation produced significant overdispersion in events of plasticity compared with Poisson $\left(\chi^{2} p<0.0001\right)$, the spaced stimulation did not $\left(\chi^{2} p=\right.$ $0.5)$ (Lawless, 1987) (Fig. 4B, G,H). This means that, whereas the massed stimulation induces a variable number of new synaptic boutons in different synapses (low precision of the synaptic response), the spaced pattern always produce a similar response (high precision of the synaptic response).

In addition, the protocol of 4 pulses of $4 \mathrm{~min}$ (Fig. $2 C$ ) induced a synaptic response with intermediate properties between those produced by massed and spaced stimulation (Fig. $4 K$ ), indicating that 5 pulses are required to develop such precision in plasticity.

The synaptic response after the pseudo-massed protocol containing ISIs of $10 \mathrm{~min}$ (Fig. 2B) showed a data distribution with properties of that produced by massed stimulation (Fig. $4 L$ ), supporting that, by reducing the ISI to $10 \mathrm{~min}$, the stimulation pattern was decoded as a massed stimulation. Similarly, the synaptic response after the standard pseudo-massed protocol by reducing Ras or rolled activity (Fig. $3 A, B, D$ ) showed a data distribution with properties of that produced by massed stimulation (Fig. $4 M, N$ ), supporting that the stimulation pattern was decoded as a massed stimulation in the absence of these molecular components.

We confirmed the conclusion that the spaced stimulation induced a more precise response in synaptic plasticity using a distribution-independent analysis. We examined variability in synaptic response by resampling of the experimental data through the bootstrapping technique. We found a variance 4.10 times larger after massed than spaced stimulation (Fig. $5 A-C)$. Together, these observations suggest that the integration of spacing and repetition effects reduces imprecise synaptic plasticity responses.

Next, because precision in synaptic plasticity was higher after five than four spaced stimuli (Fig. $5 C$ ), we tested whether a larger number of pulses produce a higher precision. We examined the precision in synaptic plasticity after a protocol of 5 or 8 pulses of 2 min spaced by 15 min (totaling 10 and 16 min of stimulation) compared with 10 and 16 min of massed stimulation.

First, 10 and 16 min of massed or spaced stimulation produced a significant number of new synaptic boutons compared with the nonstimulated preparation (Fig. $5 A, B$ ). Second, stimulation with 8 pulses of 2 min generated a synaptic response as precise as the standard spaced protocol ( 5 stimuli totaling $16 \mathrm{~min}$; Fig. $5 C$ ) and produced a data distribution with similar properties to the standard spaced stimulation (Fig. $5 D, E$ ). This confirmed that the spaced protocol produces higher precision in synaptic plasticity. However, 5 pulses of 2 min (totaling $10 \mathrm{~min}$ of stimulation) produced a synaptic response as variable as $10 \mathrm{~min}$ of massed stimulation (Fig. $5 \mathrm{C}$ ) with properties in data distribution compatible with massed stimulation (Fig. $5 F$ ). This result allows us to conclude that, in addition to the number of stimuli (five pulses), the total time of stimulation in a spaced protocol is also important for precision in structural synaptic plasticity.

Synaptic plasticity was prevented by ISI-dependent inhibition in the pseudo-massed protocol, suggesting that this inhibitory 
A

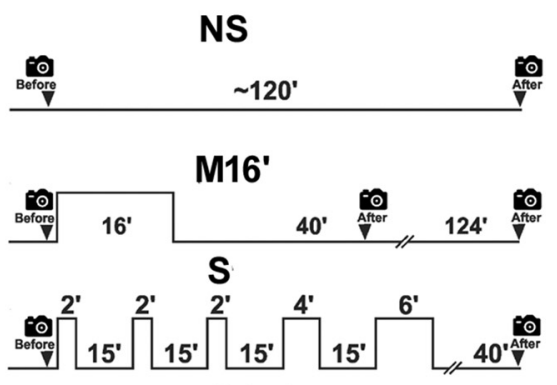

$54 \times 4$

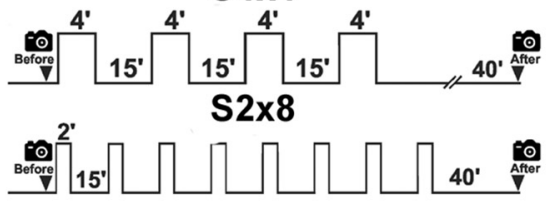

S2x5
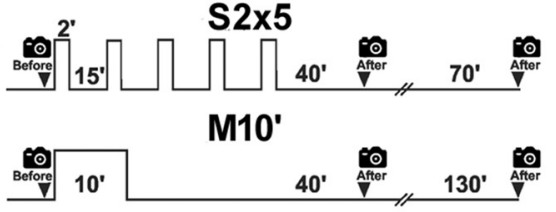

B

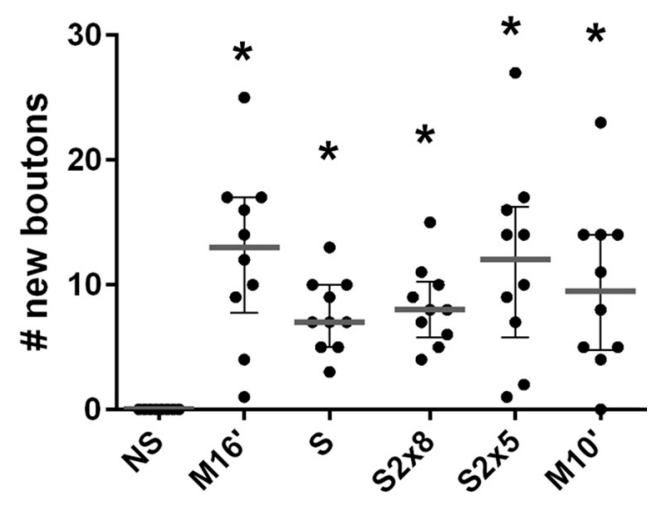

D

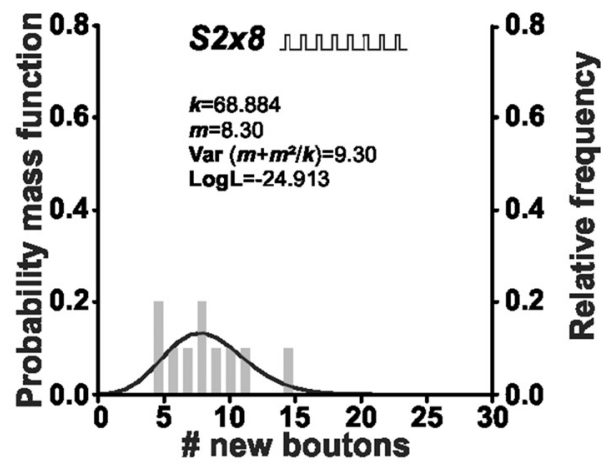

$\mathbf{F}$

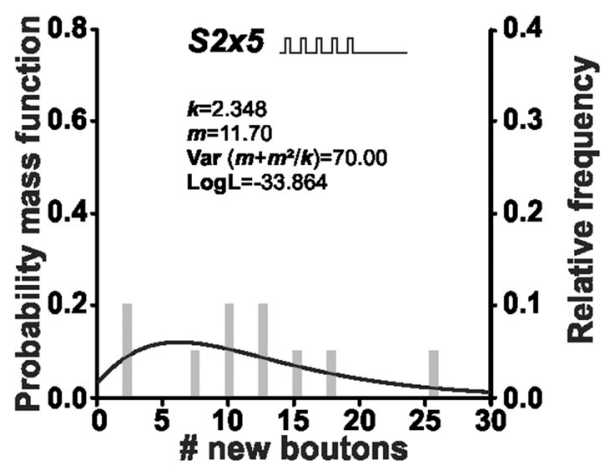

Figure 5. Precision in structural synaptic plasticity requires prolong spaced stimulation. A, Protocols of stimulation: control nonstimulated (NS), massed stimulation of 16 min (M16'), spaced stimulation (S), symmetric stimulation (S4×4), spaced stimulation composed of 8 pulses of 2 min spaced by $15 \min (S 2 \times 8)$, spaced stimulation composed of 5 pulses of 2 min spaced by 15 min $(S 2 \times 5)$ and massed stimulation of $10 \mathrm{~min}\left(\mathrm{M} 10^{\prime}\right)$. B, Quantification of new synaptic boutons $40 \mathrm{~min}$ after stimulation (schematized in $\boldsymbol{A}$, except $S 4 \times 4$ shown in Fig. 2C). Scatter dot plot showing median (gray line) and interquartile range. $n=10 \mathrm{NMJs}$ from 5 animals. *Significant difference compared with NS, M16', $p<0.0001 ; S, p=0.0123 ; S 2 \times 8, p=0.0043 ; S 2 \times 5, p<0.0001 ;$ and M10', $p=0.0014$, Kruskal-Wallis test, followed by Dunn's multiple-comparisons test. C, Mean variance obtained by resampling $n=10,000$ through the bootstrapping technique after stimulation (schematized in $A$ ). Error bars indicate mean variance $\pm S E M$. Asterisks indicate reduced variance: M16' versus $S, p=0.0012 ; \mathrm{M} 16^{\prime}$ versus $S 2 \times 8, p=0.006 ; S$ vs $S 4 \times 4, p=0.0293$, Monte Carlo permutation test. $\boldsymbol{D}$, Fits of the negative binomial distribution to the observed new synaptic boutons after stimulation $S 2 \times 8, n=10 \mathrm{NMJs}$ from 5 animals. $\boldsymbol{E}$, New synaptic boutons versus number of pulses in protocols totaling $16 \mathrm{~min}$ of stimulation. Exponential one phase decay fit for three or more pulses. $n=10 \mathrm{NMJs}$ from 5 animals for each number of pulses. $\boldsymbol{F}$, Fits of the negative binomial distribution to the observed new synaptic boutons after stimulation $\$ 2 \times 5, n=10 \mathrm{NMJs}$ from 5 animals. 
A

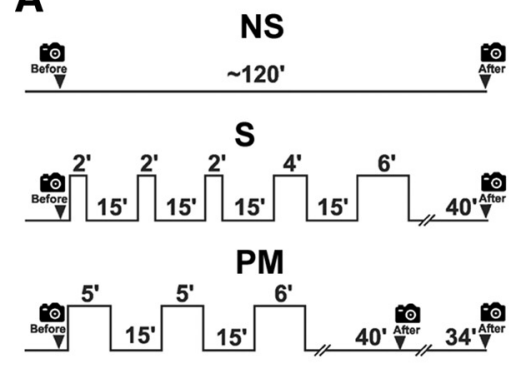

B

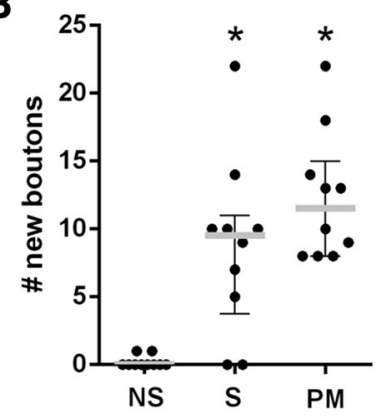

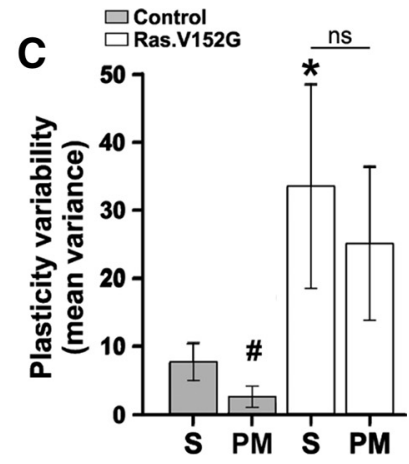

D

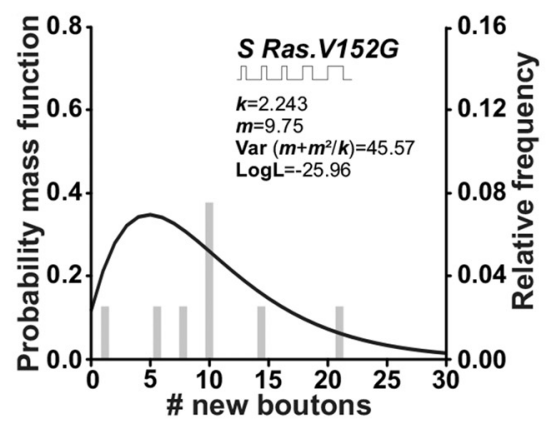

E

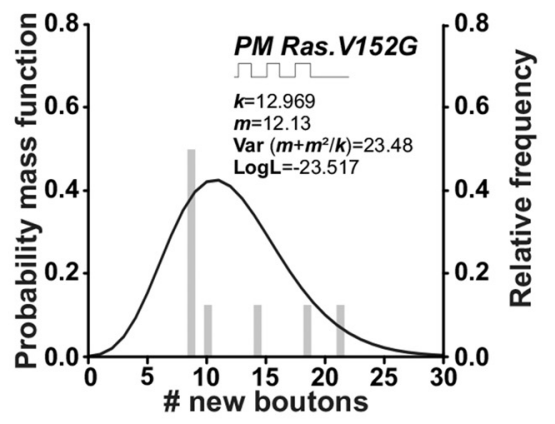

Figure 6. Ras GOF mutation impairs specificity and precision of synaptic response. $A$, Protocols of stimulation: control nonstimulated (NS), spaced (S), and pseudo-massed (PM). B, Quantification of new synaptic boutons 40 min after stimulation (schematized in $A$ ) in motoneurons expressing the GOF Ras.V152G mutant allele. Scatter dot plot showing median (gray line) with interquartile range. $n=10 \mathrm{NMJs}$ from 5 animals. *Significant plasticity compared with NS, S, $p=0.008 ; \mathrm{PM}, p=0.001$, Kruskal-Wallis test followed by Dunn's multiple-comparisons test. C, Mean variance obtained by resampling $n=10,000$ through the bootstrapping technique after different protocols of stimulation: $S$ and PM. Error bars indicate mean variance \pm SEM. *Larger variance compared with the $S$ protocol in the control genotype, $p=0.0177$, Monte Carlo permutation test. \#In the control genotype, PM stimulation does not induced structural plasticity. $\boldsymbol{D}, \boldsymbol{E}$, Fits of the negative binomial distribution to the observed new synaptic boutons in GOF Ras.V152G after $S(\boldsymbol{D})$ and PM stimulation $(\boldsymbol{E}), n=10 \mathrm{NMJs}$ from 5 animals.

effect prevents plasticity after a short stimulation, which can be expected to be imprecise. Moreover, the integration of the spacing and the repetition effects occurring in the spaced protocol (five or more stimuli totaling $16 \mathrm{~min}$ ) produced more precise synaptic plasticity compared with the same amount of stimulation in a massed pattern.

Ras gain-of-function (GOF) mutations impair specificity and precision in synaptic plasticity

A group of disorders known as RASopathies are caused by GOF mutations of various genes including Ras. The resulting mutant proteins show higher and more persistent activity affecting the spacing effect for memory, in contrast to the overexpression of WT proteins that only show higher activity (Costa et al., 2002; Li et al., 2005; Cui et al., 2008; Pagani et al., 2009; San Martín and Pagani, 2014). Such persistent activity in different GOF mutations was more prolonged than the activity required during the 15 min of intertrial interval producing LTM impairment (Pagani et al., 2009). At the synaptic level, the spacing effect for structural plasticity required $>10$ min of ISI (Fig. $2 B$ ) and depended on Ras signaling (Fig. 3). Therefore, the data presented above (Figs. 2, 3, $4,5)$ predict that Ras GOF mutants would be unable to induce synaptic plasticity normally because of a persistent activation (more prolonged than that normally required for the $15 \mathrm{~min}$ of ISI). In other words, the expression of Ras GOF might produce a similar effect to the shortening of the ISI to $10 \mathrm{~min}$ (Fig. $2 \mathrm{~B}, L$ ). Therefore, any stimulation pattern containing spaced stimuli might be decoded as a massed stimulation in these mutants. To test this possibility, we examined the effect of the GOF Ras allele
V152G (Carta et al., 2006) on plasticity after spaced and pseudomassed stimulation. As expected, the specificity of the synaptic response to the spaced and pseudo-massed protocol was lost because both stimulation patterns produced a large number of new synaptic boutons (Fig. 6A, B). Furthermore, the precision in the synaptic response was also lost because the spaced and pseudomassed stimulation induced a highly variable response in plasticity compared with the control genotype (Fig. 6C). Finally, the data produced by spaced and pseudo-massed stimulation in the RasV152G mutant showed a distribution compatible with the data produced by massed stimulation in the control genotype, low $k$ value and high variance (Fig. $6 D, E$ ), supporting the notion that in this Ras mutant spaced stimuli are decodes as massed stimulation.

Because the spacing effect was lost with the GOF Ras mutant, our experiments suggest a pathological alteration in how synapses decode stimulus patterns for activity-dependent structural synaptic plasticity. More importantly, these experiments provide evidence for a model in which the integration of the spacing and the repetition effects constitute a mechanism for disambiguation in synaptic plasticity in which Ras is required.

\section{Spaced, but not massed, stimulation potentiates mEJP frequency}

Spaced stimulation potentiates mEJP frequency at the NMJ through a retrograde synaptotagmin 4-dependent signaling mechanism (Yoshihara et al., 2005; Ataman et al., 2008; Korkut et al., 2013). In addition, mEJP, but not evoked EJP, seems to be required for developmental synapse growth (Choi et al., 2014). Therefore, we 
investigated whether the stimulation patterns that produce structural synaptic plasticity (Fig. $1 A, D$ ) also potentiate mEJP frequency. To test this possibility, we provided the stimulation patterns as before (Fig. 1A) and then examined mEJP frequency as described previously (Ataman et al., 2008). In contrast to our expectations, the spaced protocol potentiated mEJP frequency compared with the nonstimulated preparation, whereas massed or pseudo-massed stimulation did not (Fig. 7A,B). Moreover, mEJP frequency showed a linear regression with the number of pulses (Fig. 7C). Therefore, functional and structural synaptic plasticity did not correlate in these conditions. Because 16 min of spaced, but not massed, stimulation potentiated mEJP frequency, these observations show the existence of a spacing effect for functional synaptic plasticity.

\section{Ras manipulations preclude crawling-dependent} synaptic growth

The rapid formation of ghost boutons shares some of the mechanisms of developmental bouton formation (Korkut et al., 2013; Piccioli and Littleton, 2014). Therefore, our observations might predict that Ras manipulation produces structural defects in the developmentally formed boutons and in turn behavioral defects in locomotor activity. Because the pseudo-massed stimulation induced new synaptic boutons in Ras.N17, Ras.RNAi, and rl.RNAi animals, but not in the control genotype (Fig. 3), inhibition of Ras/MAPK seems to facilitate structural plasticity. Moreover, overexpression of Ras.WT precluded synaptic bouton formation even after the spaced stimulation (Fig. 3). Together, these data suggested that Ras might work similarly to the focal adhesion kinase (Fak56), which suppresses crawling-activitydependent NMJ growth (Tsai et al., 2008, 2012). To explore this possibility, we cultured fruit flies at $29^{\circ} \mathrm{C}$ as described previously (Tsai et al., 2012), which induced temperature-dependent high crawling activity and in turn promoted the formation of synaptic boutons (synapses growth). Then, we examined the relation between crawling activity and synapse growth in larvae expressing Ras transgenes used above (Figs. 3, 6). Crawling levels in animals expressing Ras.N17, Ras.RNAi, or RasV152G in motoneurons were indistinguishable from the control genotype $(+/+$;CD8.mRFP; $D 42 /+$ ) (Fig. $8 A, B$ ). In contrast, overexpression of Ras.WT enhanced crawling activity (Fig. $8 A, B$ ). We also investigated whether such levels of activity promote a consistent degree of synapse growth, measured as total number of synaptic boutons. Control animals showed a high number of synaptic boutons compared with any of the animals with Ras manipulation (Fig. $8 C$ ). Therefore, these results suggest that Ras manipulations preclude crawling-dependent synaptic growth even after a higher crawling in animals overexpressing Ras.WT.

\section{Discussion}

We investigated the production and distribution of discrete events of structural synaptic plasticity induced by spaced stimuli at the single synapse level. Our study provides evidence that synapses compute and integrate spaced stimuli differently depending on the number of pulses, the length of the intervals between pulses, and the total time of stimulation.

We found that the spacing effect is a phenomenon detected at the synaptic level, which determines mEJP frequency potentiation and the specificity and precision in structural synaptic plasticity as well.

An additional important finding in our investigations is that the spacing in a short protocol (three pulses) inhibits synaptic plasticity (Figs. 1A-D, 2B) (Wu et al., 2001; Ataman et al., 2008).
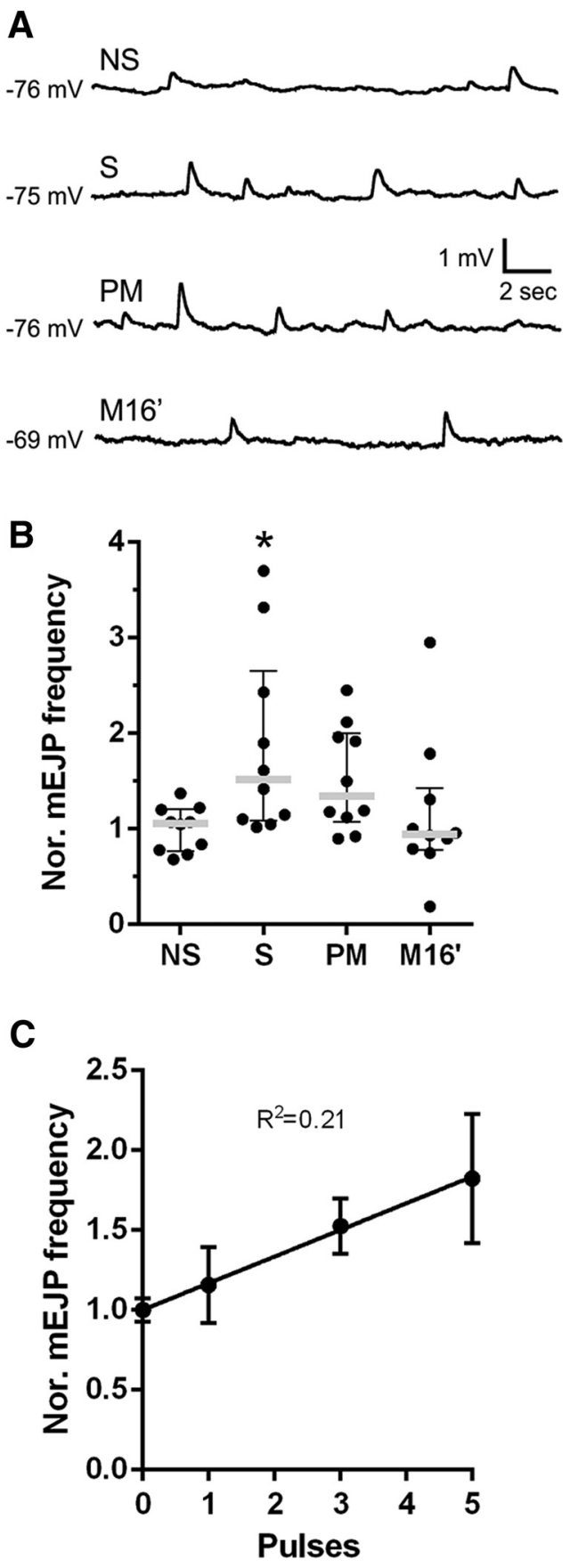

Figure 7. Spaced, but not massed, stimulation potentiates mEJP frequency. $\boldsymbol{A}$, Representative recordings of $\mathrm{mEJPs}$ from a nonstimulated (NS) preparation and from preparations subjected to spaced (S), pseudo-massed (PM), or massed (M16') stimulation in NMJ 6/7 of control genotype (CD8.mRFP; D42/+). B , Frequency of mEJPs recorded from fibers 6/7, $15 \mathrm{~min}$ after each of the treatments shown in $\boldsymbol{A}$ normalized to the average frequency observed in nonstimulated preparations. Scatter dot plot showing median (gray line) with interquartile range. Black dots represent each replicate. $n=10$ NMJs from 5 animals. *Significant difference compared with control NS; S, $p=0.0367$, Kruskal-Wallis test followed by Dunn's multiple-comparisons test. $C$, Average frequency of mEJP versus number of pulses in protocols totaling $16 \mathrm{~min}$ of stimulation showing a linear relationship, $p=0.0027, n=10$ NMJs from 5 animals for each number of pulses.

The lack of plasticity in this protocol was avoided when the spacing was shortened to 10 min (Fig. 2B) (Vasin et al., 2014). Consistent with this, whereas suppressing Ras activity by Ras.N17 or Ras.RNA $i$ allowed us to detect synaptic plasticity after pseudomassed stimulation (Fig. $3 A, B$ ), overexpressing Ras-WT pre- 
A
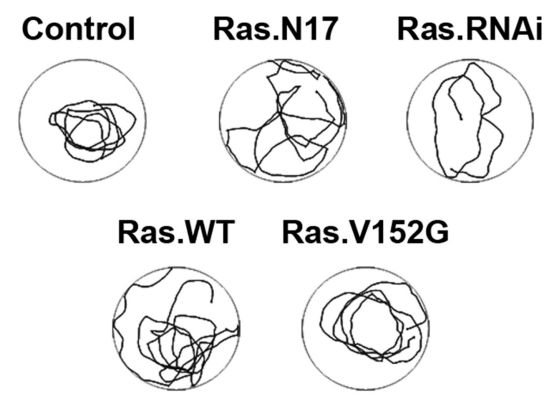

B

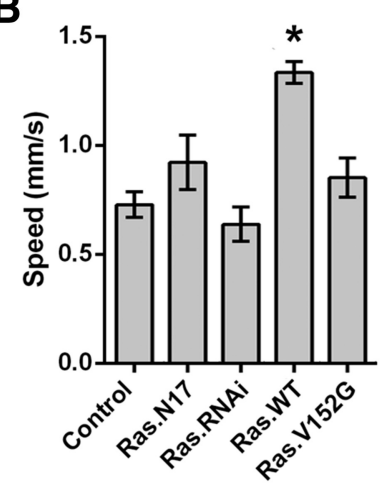

C

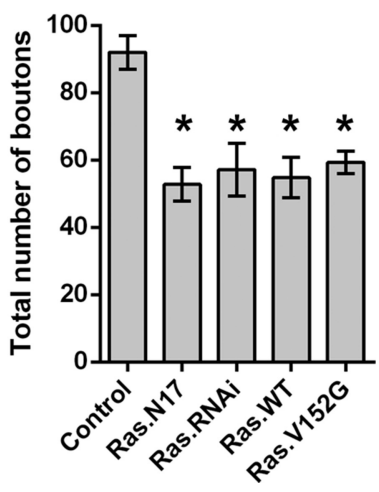

Figure 8. Ras manipulations preclude crawling-dependent synaptic growth. $\boldsymbol{A}$, Representative tracking of the crawling assay for larvae expressing Ras alleles in motoneurons and control genotype. B, Overall speed, calculated as distance traveled over mobility time, for the different genotypes. $n=7$ animals. * Significant differences compared with control genotype speed, $p<$ 0,0001 , ANOVA test followed by Dunn's multiple-comparisons test. $C$, Total number of boutons of the NMJ $6 / 7$ in third instar larvae raised at $29^{\circ} \mathrm{C} . n=6 \mathrm{NMJs}$ from 6 animals. ${ }^{*}$ Significant differences compared with control genotype, Ras.N17, $p=0.0002$; Ras.WT, $p=0.0004$; Ras.V152G, $p=0.0014$; Ras.RNAi, $p=0,0007$, AN0VA test followed by Dunn's multiple-comparisons test.

vented plasticity after the standard protocol of spaced stimuli (Fig. 3C). Inhibition of MAPK using a rolled-RNAi showed a similar effect to the inhibition of Ras (Fig. 3D). These genetic manipulations of Ras or MAPK had no effect on the synaptic plasticity after massed stimulation (Fig. 3E), supporting the idea that spacing is required for these Ras/MAPK-dependent effects (Fig. 3). Therefore, genetic manipulations of Ras/MAPK changed how a synapse decodes the information of a stimulation pattern (Figs. 1, 3).

In addition, a GOF mutation of Ras (i.e., V152G) impaired the specificity of the synaptic response and the precision in synaptic plasticity induced by spaced stimuli (Fig. 6A,B). This is a straightforward interpretation because Ras GOF induced synaptic plasticity after the spaced and pseudo-massed stimulation with a highly variable number of new synaptic boutons (Fig. $6 C-E)$. The plasticity induced by pseudo-massed stimulation with the Ras GOF mutant can be explained by an enhanced activity of Ras, which might disrupt the spacing effect for structural synaptic plasticity, making it equivalent to shortening the ISI to 10 min (Fig. $2 B$ ). In this Ras mutant, the synaptic response after any stimulation was as variable as in the control genotype after the pseudo-massed with ISIs of $10 \mathrm{~min}$ (Fig. 2B). Overexpression of Ras cannot explain the effect of the GOF allele because overexpressing Ras-WT prevented the formation of new boutons after the spaced protocol (Fig. 3C). Together, these observations suggest that the plasticity in the Ras GOF mutant is decoded as massed stimulation, which is consistent with the effect of the corkscrew GOF alleles in memory after spaced training (Pagani et al., 2009). Therefore, the spacing effect at the synaptic level seems to be essential for the specificity and the precision of the synaptic response to a stimulus pattern (Fig. 9) because, in absence of the spacing effect, the repetition effect cannot be encoded.

\section{Effect of the spacing and repetition}

Our experiments and previous reports suggest that the ISIdependent inhibition and the repetition effect work in distinct moments and by different mechanisms. Ras/MAPK signaling modulated stimulation-dependent new synaptic boutons formation, but did not participate directly in the formation because the inhibition of those components (using Ras.N17, Ras.RNAi,or rl.RNAi) did not prevent the formation of new synaptic boutons (Fig. 3). In fact, the expression of those transgenes seems to facilitate structural plasticity, in contrast to the overexpression of Ras.WT that precluded it (Fig. 3). These data are consistent with a model in which rolled, controlled by retrograde signaling, inhibits fasciclin II and in turn prevents structural plasticity (Tsai et al., 2008 and 2012). It is tempting to speculate that such retrograde mechanism somehow controls the time required for the effectiveness of the spacing effect. In addition, Ras/MAPK appear to exert their effect during the ISI because, in absence of ISIs, there was no significant effect on plasticity (Fig. 3D).

Moreover, the timing in which new synaptic boutons came out suggests that the repetition exerts its effect during the pulse. In Drosophila NMJ, it was shown previously that, immediately after three pulses of stimulation (spaced by $15 \mathrm{~min}$ ), there was no new synaptic boutons. However, new synaptic boutons were detected immediately after the fourth pulse of stimulation and persisted at least up to $130 \mathrm{~min}$ after stimulation (Ataman et al., 2008). New boutons appear only during the pulse of $\mathrm{K}^{+}$stimulation, but not during the ISIs in the Drosophila NMJ (Piccioli and Littleton, 2014). A similar phenomenon was found in cultured hippocampal neurons stimulated with $\mathrm{K}^{+}$(Wu et al., 2001). Together, these data suggest that repetition and ISI-dependent inhibition work, respectively, during the pulse of stimulation and the intervals and, presumably, they are integrated along the course of the stimulation protocol.

\section{Spaced stimuli promote precision in structural synaptic plasticity}

Count data are usually not distributed randomly, providing information about the distribution of the underlying mechanisms (Bliss and Fisher, 1953). This was the case after massed stimulation, which showed overdispersion; however, the spaced stimulation promoted a random distribution (Poisson process; Fig. 4H; Bliss and Fisher, 1953; Lindén and Mäntyniemi, 2011). The main difference in data sampling was a greater manipulation during spaced stimulation, which should provide a larger variation, but not smaller (Fig. $4 G, H$ ). Therefore, the distributions observed might reflect the distribution of the underlying mechanisms, for instance, the rate of relocation and nanoclustering of Ras within the nerve terminal after pulses of stimulation, which in turn regulates synaptic bouton formation (Harvey et al., 2008; Murakoshi et al., 2011; Murakoshi and Yasuda, 2012; Zhou et al., 2015).

We detected structural synaptic plasticity after massed stimulation (Fig. 1). In contrast, a previous report did not detect plastic changes after a similar stimulation procedure in cultured hippocampal neurons (Wu et al., 2001). One possible 


$$
\begin{aligned}
& \text {-No ISI } \\
& \text {-No Repetition }
\end{aligned}
$$
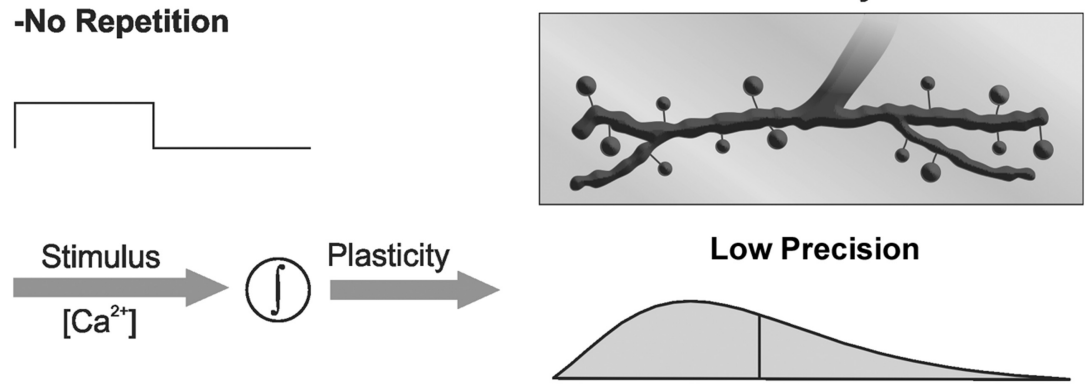

Low Precision

$\mathrm{Ca}^{2+}$

\section{-ISI}

-Low Repetition

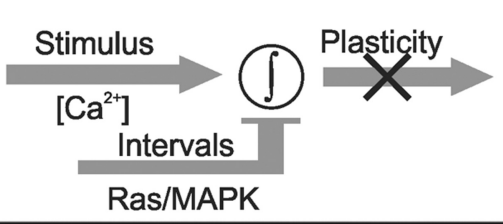

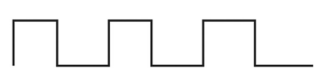
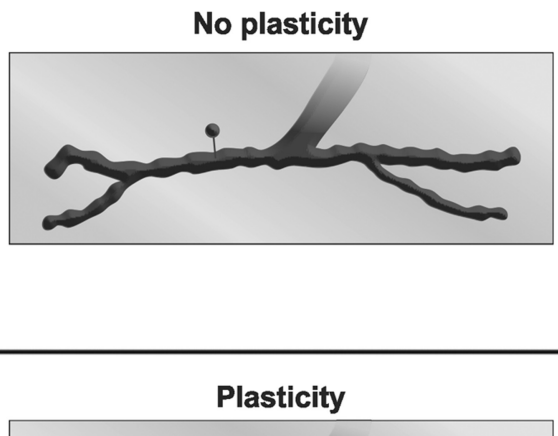

$-\mid \mathbf{S I}$

-High Repetition
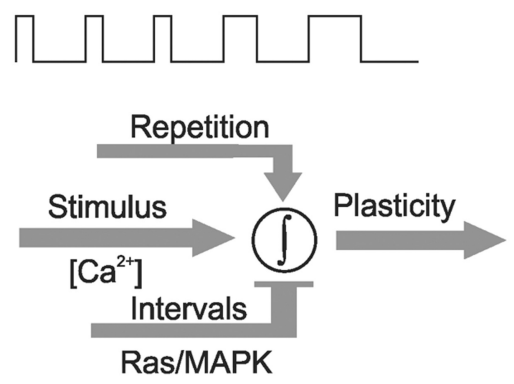

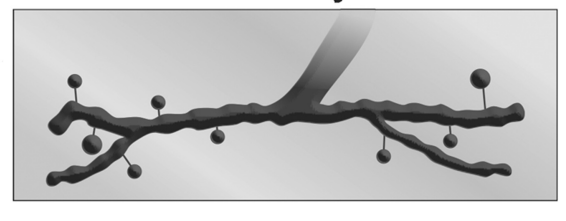

High Precision

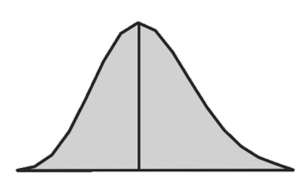

Figure 9. Schematic model summarizing the properties of three distinct patterns of stimulation and their specificity in structural synaptic plasticity response. Three patterns of stimuli with the same total time of stimulation showed different responses depending on the number of stimuli and ISIs of $15 \mathrm{~min}$. The synaptic response is $\mathrm{Ca}^{2+}$-and activity-dependent new synaptic boutons (Ataman et al., 2008). Top, One long pulse with no ISI produced a significant response in structural synaptic plasticity (boutons), but this response is highly variable. Middle, Three spaced pulses did not produce plasticity as a result of a Ras/MAPKdependent inhibition acting during the ISIs. Bottom, If the number of pulses is increased $(5 \times)$, then the inhibition mediated by Ras/MAPK is overcome and the synaptic response is significant and more precise.

explanation is that synaptic plasticity after massed stimulation is only observable at certain synapses, including the larval NMJ. Alternatively, it is also possible that the hippocampal explants used after 3-4 weeks of culture (Wu et al., 2001) had no completely functional synaptic connections lacking part of the retrograde mechanisms required for normal synaptic function and plasticity (Yoshihara et al., 2005; Dean et al., 2009; Korkut et al., 2013). This may explain why the investigators only detected formation and enlargement of filopodia, not dendritic spines, after spaced stimulation (Wu et al., 2001).

Lack of plasticity after three pulses of stimulation, regardless of the duration of each pulse, as well as the ability to produce it after four pulses (spaced by $15 \mathrm{~min}$ ), was detected previously in cultured hippocampal neurons and in fruit fly NMJ (Wu et al., 2001; Ataman et al., 2008), predicting that this may be a general phenomenon not limited to our experimental model or conditions. Spaced, but not massed, stimulation produced a more precise response in structural plasticity, which in turn might have important implications because neuronal connectivity determines brain function. Spaced stimuli involve a system in which a given time of activity (e.g., $16 \mathrm{~min}$ ) is more accurately decoded. This property can be paramount in many processes considering that the information is not carried by a single neuron, but through at least several neurons in parallel (e.g., from dentate gyrus to CA 3 in the hippocampus). For instance, a precise synaptic response in structural plasticity after a specific experience or activity pattern may ensure a specific balance between excitatory and inhibitory inputs or that neurons operating in parallel maintain a similar synaptic strength, as suggested for parallel synapses within an axonal branch (Bartol et al., 2015). Moreover, 16 min of spaced, but not massed, stimulation potentiates $\mathrm{mEJP}$ frequency (Fig. 7), which in turn may regulate synapse growth (Choi et al., 2014).

One important prediction of our observations is that a higher precision in synaptic plasticity may also underlie the spacing effect for memory. Remarkably, in cerebellum-dependent motor learning, the spaced training produced several times fewer variation in synaptic density compared with the massed training (Aziz et al., 2014). If such higher precision in synaptic plasticity is involved in spaceddependent learning, we can speculate about its behavioral implications. One possibility is that the resulting behavioral performance also would be more precise after the spaced training. Although interesting, such a situation was found in few (Pagani et al., 2009; Aziz et al., 2014), but not most, cases in the literature (Tully et al., 1994; Scharf et al., 2002; Jackson et al., 2013; Zhao et al., 2013). Alternatively, a more precise response in structural synaptic plasticity might contribute to a more persistent memory, as previous observations suggest (Aziz et al., 2014). Regardless of the precision, spaced training provides a more enduring structural plasticity than the massed one, which correlates with the persistence of the resulting memory (Aziz et al., 2014).

Previous studies have proposed that molecular mechanisms involving the Ras/MAPK signaling pathway and the coordination of this pathway with PKA signaling may underlie the spacing effect for memory (Pagani et al., 2009; Zhang et al., 2011; Smolen et al., 2016). It should be noted that genetic and pharmacological manipulations of Ras/MAPK signaling pathway impair LTP and consolidated memories after spaced training, but not after massed training (Pagani et al., 2009; Smolen et al., 2016). Here, our experiments suggest that similar molecular signaling pathways may contribute to decode patterns of activity determining the response in structural synaptic plasticity, which in turn would support the spacing effect for memory. 


\section{Ras in structural synaptic plasticity}

Motoneurons expressing a Ras GOF allele lose the Ras-dependent inhibition in the pseudo-massed protocol, as well as a precise synaptic response, after the standard spaced stimulation. The fact that an ISI of 10 min prevented the inhibitory effect indicates that Ras requires a longer time than that to produce its effect. Therefore, Ras appears to be acting as a detector of stimuli determining whether two pulses are separated enough to be computed as different stimuli or just a single one.

Growth of single dendritic spines induced by glutamate uncaging in cultured hippocampal neurons requires $\mathrm{Ca}^{2+}$ influx and subsequent activation of CaMKII, calcineurin, Ras, RhoA, and Cdc42 (Lee et al., 2009; Murakoshi et al., 2011; Murakoshi and Yasuda, 2012). Extracellular calcium is also required for the plastic changes examined in our study (Ataman et al., 2008). Whereas the fast $\mathrm{Ca}^{2+}$ signal lasts $\sim 0.1 \mathrm{~s}$, downstream protein activation expands the time scale from seconds to $\sim 1 \mathrm{~min}$ in the case of CaMKII, which in turn activates small G-proteins lasting several minutes (Murakoshi and Yasuda, 2012). Our study showed that Ras regulates synaptic plasticity, but requires $>10$ min of ISI to be effective (Figs. 2, 3). Calcineurin activation responds mainly to stimulus number, whereas CaMKII responds to high number and frequency of stimuli. However, in both cases, a significant response required a minimum stimulation frequency of $2 \mathrm{~Hz}$ (Fujii et al., 2013). This requirement indicates that such computation of stimulus required the integration of molecular events initiated by successive pulses of stimulation. However, the time scale in our experiments is much larger than those previous reports (Harvey et al., 2008; Lee et al., 2009; Murakoshi et al., 2011; Murakoshi and Yasuda, 2012; Fujii et al., 2013). Therefore, our studies explored mechanisms involved in plastic changes subsequent to those reported previously (Harvey et al., 2008; Fujii et al., 2013). Plasticity at larger timescale was shown by studies of LTP-mediated TBS, in which two TBS spaced by 60 $\mathrm{min}$, but not $30 \mathrm{~min}$, induced a larger number of dendritic spines compared with a single TBS (Kramár et al., 2012).

In hippocampal neurons, Ras is necessary for sustained, but not for transient, structural LTP (Harvey et al., 2008). However, hyperactivation of Ras by suppressing NF1 activity results in spine loss in hippocampal neurons because of sustained Ras activity (Oliveira and Yasuda, 2014). Likewise, increasing Ras-WT activity prevented synaptic plasticity after spaced stimuli (Fig. $3 C$ ). Therefore, the role of Ras in structural plasticity was documented in different neuronal types and distinct animal models or preparations. Together, our experiments support that the spacing effect and Ras seem to be widespread mechanistic components in the nervous system for generating plastic changes.

\section{Notes}

Supplemental material for this article is available at http://www.ifibio-ubaconicet.gob.ar/wp-content/uploads/2016/05/Supplemental-Materials-1 -4.pdf. Results presented herein as "data not shown" are available on our website as supplementary material. This material has not been peer reviewed.

\section{References}

Ataman B, Ashley J, Gorczyca M, Ramachandran P, Fouquet W, Sigrist SJ, Budnik V (2008) Rapid activity-dependent modifications in synaptic structure and function require bidirectional Wnt signaling. Neuron 57: 705-718. CrossRef Medline

Aziz W, Wang W, Kesaf S, Mohamed AA, Fukazawa Y, Shigemoto R (2014) Distinct kinetics of synaptic structural plasticity, memory formation, and memory decay in massed and spaced learning. Proc Natl Acad Sci U S A 111:E194-E202. CrossRef Medline

Bailey CH, Chen M (1988) Long-term sensitization in Aplysia increases the number of presynaptic contacts onto the identified gill motor neuron L7. Proc Natl Acad Sci U S A 85:9356-9359. CrossRef Medline

Bailey CH, Chen M (1989) Time course of structural changes at identified sensory neuron synapses during long-term sensitization in Aplysia. J Neurosci 9:1774-1780. Medline

Bailey CH, Montarolo P, Chen M, Kandel ER, Schacher S (1992) Inhibitors of protein and RNA synthesis block structural changes that accompany long-term heterosynaptic plasticity in Aplysia. Neuron 9:749-758. CrossRef Medline

Bartol TM, Bromer C, Kinney J, Chirillo MA, Bourne JN, Harris KM, Sejnowski TJ (2015) Nanoconnectomic upper bound on the variability of synaptic plasticity. Elife 4.

Beck CD, Schroeder B, Davis RL (2000) Learning performance of normal and mutant Drosophila after repeated conditioning trials with discrete stimuli. J Neurosci 20:2944-2953. Medline

Biggs WH 3rd, Zavitz KH, Dickson B, van der Straten A, Brunner D, Hafen E, Zipursky SL (1994) The Drosophila rolled locus encodes a MAP kinase required in the sevenless signal transduction pathway. EMBO J 13:16281635. Medline

Bliss CI, Fisher RA (1953) Fitting the negative binomial distribution to biological data. Biometrics 9:176. CrossRef

Brunelli M, Castellucci V, Kandel ER (1976) Synaptic facilitation and behavioral sensitization in Aplysia: possible role of serotonin and cyclic AMP. Science 194:1178-1181. CrossRef Medline

Carew TJ, Pinsker HM, Kandel ER (1972) Long-term habituation of a defensive withdrawal reflex in Aplysia. Science 175:451-454. CrossRef Medline

Carta C, Pantaleoni F, Bocchinfuso G, Stella L, Vasta I, Sarkozy A, Digilio C, Palleschi A, Pizzuti A, Grammatico P, Zampino G, Dallapiccola B, Gelb BD, Tartaglia M (2006) Germline missense mutations affecting KRAS Isoform B are associated with a severe Noonan syndrome phenotype. Am J Hum Genet 79:129-135. CrossRef Medline

Castellucci VF, Nairn A, Greengard P, Schwartz JH, Kandel ER (1982) Inhibitor of adenosine $3^{\prime}: 5^{\prime}$-monophosphate-dependent protein kinase blocks presynaptic facilitation in Aplysia. J Neurosci 2:1673-1681. Medline

Cedar H, Kandel ER, Schwartz JH (1972) Cyclic adenosine monophosphate in the nervous system of Aplysia californica. I. Increased synthesis in response to synaptic stimulation. J Gen Physiol 60:558-569. CrossRef Medline

Cepeda NJ, Pashler H, Vul E, Wixted JT, Rohrer D (2006) Distributed practice in verbal recall tasks: a review and quantitative synthesis. Psychol Bull 132:354-380. CrossRef Medline

Choi BJ, Imlach WL, Jiao W, Wolfram V, Wu Y, Grbic M, Cela C, Baines RA, Nitabach MN, McCabe BD (2014) Miniature neurotransmission regulates Drosophila synaptic structural maturation. Neuron 82:618-634. CrossRef Medline

Costa RM, Federov NB, Kogan JH, Murphy GG, Stern J, Ohno M, Kucherlapati R, Jacks T, Silva AJ (2002) Mechanism for the learning deficits in a mouse model of neurofibromatosis type 1. Nature 415:526-530. CrossRef Medline

Cui Y, Costa RM, Murphy GG, Elgersma Y, Zhu Y, Gutmann DH, Parada LF, Mody I, Silva AJ (2008) Neurofibromin regulation of ERK signaling modulates GABA release and learning. Cell 135:549-560. CrossRef Medline

Dean C, Liu H, Dunning FM, Chang PY, Jackson MB, Chapman ER (2009) Synaptotagmin-IV modulates synaptic function and long-term potentiation by regulating BDNF release. Nat Neurosci 12:767-776. CrossRef Medline

Ebbinghaus HE (1885) Memory: a contribution to experimental psychology. New York: Dover.

Fujii H, Inoue M, Okuno H, Sano Y, Takemoto-Kimura S, Kitamura K, Kano M, ${ }^{\star *}$ Bito H (2013) Report nonlinear decoding and asymmetric representation of neuronal input information by CaMKII $\alpha$ and calcineurin. Cell Rep 3:978-987. CrossRef Medline

Gerber B, Wüstenberg D, Schütz A, Menzel R (1998) Temporal determinants of olfactory long-term retention in honeybee classical conditioning: nonmonotonous effects of the training trial interval. Neurobiol Learn Mem 69:71-78. CrossRef Medline

Harvey CD, Yasuda R, Zhong H, Svoboda K (2008) The spread of Ras activity triggered by activation of a single dendritic spine. Science 321:136140. CrossRef Medline

Hermitte G, Pedreira ME, Tomsic D, Maldonado H (1999) Context shift 
and protein synthesis inhibition disrupt long-term habituation after spaced, but not massed, training in the crab Chasmagnathus. Neurobiol Learn Mem 71:34-49. CrossRef Medline

Jackson CE, Maruff PT, Snyder PJ (2013) Massed versus spaced visuospatial memory in cognitively healthy young and older adults. Alzheimers Dement 9:S32-S38. CrossRef Medline

Kandel ER, Brunelli M, Byrne J, Castellucci V (1976) A common presynaptic locus for the synaptic changes underlying short-term habituation and sensitization of the gill-withdrawal reflex in Aplysia. Cold Spring Harb Symp Quant Biol 40:465-482. CrossRef Medline

Koh YH, Ruiz-Canada C, Gorczyca M, Budnik V (2002) The Ras1-mitogenactivated protein kinase signal transduction pathway regulates synaptic plasticity through fasciclin II-mediated cell adhesion. J Neurosci 22: 2496-2504. Medline

Koon AC, Ashley J, Barria R, DasGupta S, Brain R, Waddell S, Alkema MJ, Budnik V (2011) Autoregulatory and paracrine control of synaptic and behavioral plasticity by octopaminergic signaling. Nat Neurosci 14:190199. CrossRef Medline

Kopec AM, Philips GT, Carew TJ (2015) Distinct growth factor families are recruited in unique spatiotemporal domains during long-term memory formation in Aplysia californica. Neuron 86:1228-1239. CrossRef Medline

Korkut C, Li Y, Koles K, Brewer C, Ashley J, Yoshihara M, Budnik V (2013) Regulation of postsynaptic retrograde signaling by presynaptic exosome release. Neuron 77:1039-1046. CrossRef Medline

Kramár EA, Babayan AH, Gavin CF, Cox CD, Jafari M, Gall CM, Rumbaugh G, Lynch G (2012) Synaptic evidence for the efficacy of spaced learning. Proc Natl Acad Sci U S A 109:5121-5126. CrossRef Medline

Lawless JF (1987) Negative binomial and mixed Poisson regression. Can J Stat 15:209-225. CrossRef

Lee SJ, Escobedo-Lozoya Y, Szatmari EM, Yasuda R (2009) Activation of CaMKII in single dendritic spines during long-term potentiation. Nature 458:299-304. CrossRef Medline

Li Q, Zhang X, Hu W, Liang X, Zhang F, Wang L, Liu ZJ, Zhong Y (2016) Importin-7 mediates memory consolidation through regulation of nuclear translocation of training-activated MAPK in Drosophila. Proc Natl Acad Sci U S A 113:3072-3077. CrossRef Medline

Li W, Cui Y, Kushner SA, Brown RA, Jentsch JD, Frankland PW, Cannon TD, Silva AJ (2005) The HMG-CoA reductase inhibitor lovastatin reverses the learning and attention deficits in a mouse model of neurofibromatosis type 1. Curr Biol 15:1961-1967. CrossRef Medline

Lindén A, Mäntyniemi S (2011) Using the negative binomial distribution to model overdispersion in ecological count data. Ecology 92:1414-1421. CrossRef Medline

Menon KP, Carrillo RA, Zinn K (2013) Development and plasticity of the Drosophila larval neuromuscular junction. Wiley Interdiscip Rev Dev Biol 2:647-670. CrossRef Medline

Murakoshi H, Yasuda R (2012) Postsynaptic signaling during plasticity of dendritic spines. Trends Neurosci 35:135-143. CrossRef Medline

Murakoshi H, Wang H, Yasuda R (2011) Local, persistent activation of Rho GTPases during plasticity of single dendritic spines. Nature 472:100-104. CrossRef Medline

Oishi K, Gaengel K, Krishnamoorthy S, Kamiya K, Kim IK, Ying H, Weber U, Perkins LA, Tartaglia M, Mlodzik M, Pick L, Gelb BD (2006) Transgenic Drosophila models of Noonan syndrome causing PTPN11 gain-offunction mutations. Hum Mol Genet 15:543-553. CrossRef Medline

Oliveira AF, Yasuda R (2014) Neurofibromin is the major ras inactivator in dendritic spines. J Neurosci 34:776-783. CrossRef Medline

Pagani MR, Oishi K, Gelb BD, Zhong Y (2009) The phosphatase SHP2 regulates the spacing effect for long-term memory induction. Cell 139:186198. CrossRef Medline

Philips GT, Tzvetkova EI, Carew TJ (2007) Transient mitogen-activated protein kinase activation is confined to a narrow temporal window required for the induction of two-trial long-term memory in Aplysia. J Neurosci 27:13701-13705. CrossRef Medline

Philips GT, Ye X, Kopec AM, Carew TJ (2013) MAPK establishes a molecular context that defines effective training patterns for long-term memory formation. J Neurosci 33:7565-7573. CrossRef Medline
Piccioli ZD, Littleton JT (2014) Retrograde BMP signaling modulates rapid activity-dependent synaptic growth via presynaptic LIM kinase regulation of cofilin. J Neurosci 34:4371-4381. CrossRef Medline

Salazar C, Brümmer A, Alberghina L, Höfer T (2010) Timing control in regulatory networks by multisite protein modifications. Trends Cell Biol 20:634-641. CrossRef Medline

San Martín A, Pagani MR (2014) Understanding intellectual disability through RASopathies. J Physiol Paris 108:232-239. CrossRef Medline

Sanyal S (2009) Genomic mapping and expression patterns of C380, OK6 and D42 enhancer trap lines in the larval nervous system of Drosophila. Gene Expr Patterns 9:371-380. CrossRef Medline

Scharf MT, Woo NH, Lattal KM, Young JZ, Nguyen PV, Abel T (2002) Protein synthesis is required for the enhancement of long-term potentiation and long-term memory by spaced training. J Neurophysiol 87 2770-2777. Medline

Smolen P, Zhang Y, Byrne JH (2016) The right time to learn: mechanisms and optimization of spaced learning. Nat Rev Neurosci 17:77-88. CrossRef Medline

Stewart BA, Atwood HL, Renger JJ, Wang J, Wu CF (1994) Improved stability of Drosophila larval neuromuscular preparations in haemolymphlike physiological solutions. J Comp Physiol A 175:179-191. CrossRef Medline

Sutton MA, Ide J, Masters SE, Carew TJ (2002) Interaction between amount and pattern of training in the induction of intermediate- and long-term memory for sensitization in Aplysia. Learn Mem 9:29-40. CrossRef Medline

Tsai PI, Kao HH, Grabbe C, Lee YT, Ghose A, Lai TT, Peng KP, Van Vactor D, Palmer RH, Chen RH, Yeh SR, Chien CT (2008) Fak56 functions downstream of integrin alphaPS3betanu and suppresses MAPK activation in neuromuscular junction growth. Neural Dev 3:26. CrossRef Medline

Tsai PI, Wang M, Kao HH, Cheng YJ, Lin YJ, Chen RH, Chien CT (2012) Activity-dependent retrograde laminin A signaling regulates synapse growth at Drosophila neuromuscular junctions. Proc Natl Acad Sci U S A 109:17699-17704. CrossRef Medline

Tully T, Preat T, Boynton SC, Del Vecchio M (1994) Genetic dissection of consolidated memory in Drosophila. Cell 79:35-47. CrossRef Medline

Urcelay GP, Wheeler DS, Miller RR (2009) Spacing extinction trials alleviates renewal and spontaneous recovery. Learn Behav 37:60-73. CrossRef Medline

Vasin A, Zueva L, Torrez C, Volfson D, Littleton JT, Bykhovskaia M (2014) Synapsin regulates activity-dependent outgrowth of synaptic boutons at the Drosophila neuromuscular junction. J Neurosci 34:10554-10563. CrossRef Medline

Vlach HA, Sandhofer CM (2012) Distributing learning over time: the spacing effect in children's acquisition and generalization of science concepts. Child Dev 83:1137-1144. CrossRef Medline

Wu GY, Deisseroth K, Tsien RW (2001) Spaced stimuli stabilize MAPK pathway activation and its effects on dendritic morphology. Nat Neurosci 4:151-158. CrossRef Medline

Xiong X, Collins CA (2012) A conditioning lesion protects axons from degeneration via the Wallenda/DLK MAP kinase signaling cascade. J Neurosci 32:610-615. CrossRef Medline

Yoshihara M, Adolfsen B, Galle KT, Littleton JT (2005) Retrograde signaling by Syt 4 induces presynaptic release and synapse-specific growth. Science 310:858-863. CrossRef Medline

Zhang Y, Liu RY, Heberton GA, Smolen P, Baxter DA, Cleary LJ, Byrne JH (2011) Computational design of enhanced learning protocols. Nat Neurosci 15:294-297. CrossRef Medline

Zhao J, Lu Y, Zhao X, Yao X, Shuai Y, Huang C, Wang L, Jeong SH, Zhong Y (2013) Dissociation of rugose-dependent short-term memory component from memory consolidation in Drosophila. Genes Brain Behav 12: 626-632. CrossRef Medline

Zhou Y, Wong CO, Cho KJ, van der Hoeven D, Liang H, Thakur DP, Luo J, Babic M, Zinsmaier KE, Zhu MX, Hu H, Venkatachalam K, Hancock JF (2015) Membrane potential modulates plasma membrane phospholipid dynamics and K-Ras signaling. Science 349:873-876. CrossRef Medline 